BNL-113214-2016-JA

File \# 94163

\title{
Pb2MnTeO6 double perovskite: an antipolar antiferromagnet
}

Maria Retuerto, Stella Skiadopoulou, Man-Rong Li, Artem M. Abakumov, Mark Croft, Alexander Ignatov, Tapati Sarkar, Brian M. Abbett, Jan Pokorný, Maxim Savinov, Dmitry Nuzhnyy, Jan Prokleška, Milinda Abeykoon, Peter W Stephens, Jason P. Hodges, Přemysl Vaněk, Craig J. Fennie, Karin M. Rabe, Stanislav Kamba and Martha Greenblatt

Submitted to Inorganic Chemistry

May 2016

Photon Sciences Department

Brookhaven National Laboratory

\author{
U.S. Department of Energy \\ USDOE Office of Science (SC), \\ Basic Energy Sciences (BES) (SC-22)
}

Notice: This manuscript has been authored by employees of Brookhaven Science Associates, LLC under Contract No. DE- SC0012704 with the U.S. Department of Energy. The publisher by accepting the manuscript for publication acknowledges that the United States Government retains a non-exclusive, paid-up, irrevocable, world-wide license to publish or reproduce the published form of this manuscript, or allow others to do so, for United States Government purposes. 


\section{DISCLAIMER}

This report was prepared as an account of work sponsored by an agency of the United States Government. Neither the United States Government nor any agency thereof, nor any of their employees, nor any of their contractors, subcontractors, or their employees, makes any warranty, express or implied, or assumes any legal liability or responsibility for the accuracy, completeness, or any third party's use or the results of such use of any information, apparatus, product, or process disclosed, or represents that its use would not infringe privately owned rights. Reference herein to any specific commercial product, process, or service by trade name, trademark, manufacturer, or otherwise, does not necessarily constitute or imply its endorsement, recommendation, or favoring by the United States Government or any agency thereof or its contractors or subcontractors. The views and opinions of authors expressed herein do not necessarily state or reflect those of the United States Government or any agency thereof. 


\section{$\mathrm{Pb}_{2} \mathrm{MnTeO}_{6}$ double perovskite: an antipolar antiferromagnet}

Maria Retuerto ${ }^{1+}$, Stella Skiadopoulou ${ }^{2}$, Man-Rong Li $^{1}$, Artem M. Abakumov ${ }^{3,4}$, Mark. Croft ${ }^{5}$, Alexander Ignatov $^{5}$, Tapati Sarkar ${ }^{1 \neq}$, Brian M. Abbett ${ }^{6}$, Jan Pokorný ${ }^{2}$, Maxim Savinov ${ }^{2}$, Dmitry Nuzhnyy ${ }^{2}$, Jan Prokleška $^{7}$, Milinda Abeykoon ${ }^{8}$, Peter W Stephens ${ }^{9}$, Jason P. Hodges ${ }^{10}$, Přemysl Vaněk ${ }^{2}$, Craig J. Fennie ${ }^{6}$, Karin M. Rabe ${ }^{5}$, Stanislav Kamba ${ }^{2}$ and Martha Greenblatt ${ }^{1^{*}}$

${ }^{1}$ Department of Chemistry and Chemical Biology, Rutgers, The State University of New Jersey, 610 Taylor Road, Piscataway, NJ 08854, USA

${ }^{2}$ Institute of Physics, The Czech Academy of Sciences, Na Slovance 2, 18221 Prague 8, Czech Republic

${ }^{3}$ EMAT, University of Antwerp, Groenenborgerlaan 171, Belgium B-2020

${ }^{4}$ Chemistry Department, Moscow State University, 119991, Moscow, Russia

${ }^{5}$ Department of Physics and Astronomy, Rutgers, The State University of New Jersey, 136 Frelinghuysen Road, Piscataway, NJ 08854, USA

${ }^{6}$ Department of Applied and Engineering Physics, Cornell University, Ithaca, New York 14853

${ }^{7}$ Department of Condensed Matter Physics, Faculty of Mathematics and Physics, Charles University, Ke Karlovu 5, 12116 Prague 2, Czech Republic

${ }^{8}$ Photon Sciences Directorate, Brookhaven National Laboratory

${ }^{9}$ Department of Physics and Astronomy, State University of New York, Stony Brook NY 11794 USA

${ }^{10}$ Spallation Neutron Source, Oak Ridge National Laboratory, Oak Ridge, TN 37831, USA 


\begin{abstract}
$\mathrm{Pb}_{2} \mathrm{MnTeO}_{6}$, a new double perovskite, has been synthesized. Its crystal structure has been determined by Synchrotron X-ray and Powder Neutron Diffraction. $\mathrm{Pb}_{2} \mathrm{MnTeO}_{6}$ is monoclinic $(I 2 / \mathrm{m})$ at room temperature with a regular arrangement of all the cations in their polyhedra. However when the temperature is lowered to $\sim 120 \mathrm{~K}$ it undergoes a phase transition from $I 2 / m$ to $C 2 / c$ structure. This transition is accompanied by a displacement of the $\mathrm{Pb}$ atoms from the center of their polyhedra due to the $6 s^{2}$ lone pair electrons, together with a surprising off-centering of $\mathrm{Mn}^{2+}\left(d^{5}\right)$ magnetic cations. This strong first order phase transition is also evidenced by specific heat, dielectric, Raman, and infrared spectroscopy measurements. The magnetic characterizations indicate an antiferromagnetic (AFM) order below $T_{\mathrm{N}} \sim 20 \mathrm{~K}$; analysis of Powder Neutron Diffraction data confirm the magnetic structure with propagation vector $\boldsymbol{k}=\left(\begin{array}{lll}0 & 1 & 0\end{array}\right)$ and collinear AFM spins. The observed jump in dielectric permittivity near $\sim 150 \mathrm{~K}$ implies possible antiferroelectric behavior, however the absence of switching suggests that $\mathrm{Pb}_{2} \mathrm{MnTeO}_{6}$ can only be antipolar. First principle calculations confirmed that the crystal and magnetic structures determined are locally stable, and that antiferroelectric switching is unlikely to be observed in $\mathrm{Pb}_{2} \mathrm{MnTeO}_{6}$.
\end{abstract}




\section{Introduction}

In the past years, $\mathrm{A}_{2} \mathrm{BB}^{\prime} \mathrm{O}_{6}$ ordered double perovskites have been widely investigated due to the huge variety of interesting physical properties, including magnetoresistance, ferroelectricity, and piezoelectricity. ${ }^{1-3}$ Also magnetoelectric behavior, where magnetism and ferroelectricity are coupled, is extensively searched for these phases, for practical applications in memories, sensors and communication. ${ }^{4,5}$ These magnetoelectric materials have the intrinsic ability to couple the electric polarization to magnetization, and vice versa, with a new degree of freedom for the potential design of conventional devices. However, there are few compounds where electric dipole and spin orders coexist, and the ones reported present very low magnetoelectric response and/or low ordering temperatures. Therefore it is a grand challenge to find materials with high magnetoelectric coupling above room temperature (RT).

To design new magnetoelectric materials, the phases must contain magnetic cations and satisfy any of the requirements for ferroelectricity: i) contain lone pair cations to produce polarization; ii) form non-centrosymmetric structures with a cation able to move from the center of its position to create an electric dipole (usually a $d^{0}$ ion with second order Jahn-Teller (SOJT) effect); iii) present a collinear, cycloidal or transverse conical magnetic structure, which induces an electric dipole; iv) undergo a transition into a charge-ordered polar state. ${ }^{6-12}$ In this work, we investigate a double perovskite with $\mathrm{Pb}^{2+}$ in the $\mathrm{A}$ site. $\mathrm{Pb}^{2+}$ has a $6 s^{2}$ lone electron pair that can be polarized along a particular direction by its off-center displacement, which results in a highly asymmetric coordination environment. This displacement is caused by the high covalency of $\mathrm{Pb}(6 s)-\mathrm{O}(2 p)^{*}$ bonds due to the hybridization of the $\mathrm{Pb} 6 s$ states with the antibonding oxygen states, which is generally considered to be the effect of a stereochemically active lone electron pair. ${ }^{13,14}$ For example, in $\mathrm{Pb}_{2} \mathrm{MnWO}_{6}$ the displacement occurs along $<100>$ and $<010>$ cubic axes, resulting in an antipolar arrangement (i.e. similar to an antiferroelectric order, but in contrast to it, it does not need to be necessarily transformed to an induced ferroelectric phase by application of an electric field). ${ }^{15}$

Pb-based double perovskites with magnetic cations in the B-sites are potentially good candidates for both magnetic and ferroelectric orderings. $\mathrm{Pb}_{2} \mathrm{FeNbO}_{6}$ and $\mathrm{Pb}_{2} \mathrm{FeTaO}_{6}$ have been extensively studied since both exhibit ferroelectric order close to RT with polarization $\left(\mathbf{P}_{\mathrm{s}}\right) \|<111>$ and antiferromagnetic (AFM) order below a Néel Temperature $\left(T_{\mathrm{N}}\right) \sim 150 \mathrm{~K} .^{16,17}$ These materials 
frequently exhibit superparamagnetic clusters even above RT, and due to the biquadratic magnetoelectric coupling $\left(\sim M^{2} P^{2}\right)$ the magnetic susceptibility exhibits anomalies at the ferroelectric phase transition temperature. ${ }^{18}$ This coupling can be very large and can even allow the switching of the ferroelectric domains by a magnetic field, as was recently demonstrated at $\mathrm{RT}$ in the solid solution between $\mathrm{PbFe}_{1-x} \mathrm{Ta}_{x} \mathrm{O}_{3}$ and $\mathrm{PbZr}_{1-x} \mathrm{Ti}_{x} \mathrm{O}_{3}{ }^{19}$ With this aim, the synthesis of $\mathrm{Pb}_{2} \mathrm{MnTeO}_{6}$ was undertaken in this investigation and we demonstrate that it exhibits an antipolar ordering below $120 \mathrm{~K}$ and AFM ordering below $20 \mathrm{~K}$.

\section{Experimental Section}

Sample Preparation and Determination of its Crystal and Magnetic Structures

$\mathrm{Pb}_{2} \mathrm{MnTeO}_{6}$ was prepared by a standard solid-state technique with reagent-grade starting materials $\mathrm{PbO}, \mathrm{MnCO}_{3}$ and $\mathrm{TeO}_{2}$. A stoichiometric mixture of the starting solids was thoroughly ground and heated in an $\mathrm{O}_{2}$ flow at $1073 \mathrm{~K}$ for 12 hours to obtain a pure sample. The product was initially characterized by Powder X-ray Diffraction (PXD) for phase identification and purity. PXD was performed in a Bruker-AXS D8 diffractometer (40 kV, $30 \mathrm{~mA})$, controlled by a DIFFRACTplus software, in Bragg-Brentano reflection geometry with $\mathrm{Cu} \mathrm{K} w, \lambda=1.5406 \AA$. For the structural refinements, Powder Neutron Diffraction (PND) data were collected at RT and $14 \mathrm{~K}$ at the POWGEN instrument in the Spallation Neutron Source in Oak Ridge National Laboratory. ${ }^{20}$ Two patterns were collected to cover $d$-spacing between 0.4 and $8.5 \AA$. The PND data were refined by the Rietveld method, with the FullProf program. ${ }^{21}$ Synchrotron Powder Diffraction (SPXD) measurements at $11 \mathrm{~K}$ and $50 \mathrm{~K}$ were made at beamlines X17A and X16C of the National Synchrotron Light Source, respectively. Those data were refined with TopasAcademic. ${ }^{22} \mathrm{X}$-ray absorption near edge spectroscopy (XANES) was collected simultaneously in both the transmission and fluorescence mode for powder samples on beam line X-19A at the Brookhaven National Synchrotron Light Source.

Magnetic, Dielectric and Heat Capacity Measurements 
Magnetization measurements were carried out in a commercial Quantum Design superconducting quantum interference device (SQUID) magnetometer MPMS5. The magnetization was measured in zero field cooled (ZFC) and field cooled (FC) conditions under a $0.1 \mathrm{~T}$ magnetic field, for temperatures ranging from 5 to $300 \mathrm{~K}$. Isothermal magnetization curves were obtained at 5 and $300 \mathrm{~K}$ under an applied magnetic field that varied from -5 to $5 \mathrm{~T}$. Differential Scanning Calorimeter (DSC) experiments were performed on a Perkin Elmer DSC 7 with liquid nitrogen cooling (93-300 K). Pyris Software (Version 11.0.3, Perkin Elmer Instruments, 2009) was used for control and evaluation. The temperature dependence of Heat Capacity $\left(C_{\mathrm{P}}\right)$ was measured with the Heat Capacity option of the Physical Property Measurement System (PPMS9, Quantum Design). The standard relaxation method was used for the measurements of low and high temperature parts. The data close to the structural transition were measured by application of large temperature pulse and independent evaluation of heating and cooling part, in order to capture the effect of hysteresis. Low-frequency $(1 \mathrm{~Hz}-1 \mathrm{MHz})$ dielectric measurements were performed between 40 and $300 \mathrm{~K}$ with a Novocontrol Alpha-A high-performance frequency analyser. Cooling and heating rate was $5 \mathrm{~K} / \mathrm{min}$. The sample was placed in a He-flow Leybold cryostat; good thermal contact was secured by He gas in the sample chamber. We attempted to measure an antiferroelectric hysteresis loop close and below $T_{\mathrm{C}}$ at frequencies from 1 to $50 \mathrm{~Hz}$ with our custom-made setup.

\section{THz, Infrared and Raman Studies}

The spectroscopic experiments were performed with a Fourier-transform infrared (IR) spectroctrometer Bruker IFS 113v equipped with a helium-cooled bolometer (operating temperature $1.6 \mathrm{~K}$ ) as a detector and a custom-made time-domain $\mathrm{THz}$ spectrometer. ${ }^{23}$ In both experiments, Optistat CF cryostats (Oxford Instruments) with polyethylene (far IR) or Mylar (THz) windows were used for the measurements between 10 and $300 \mathrm{~K}$. In the $\mathrm{THz}$ spectrometer, a femtosecond Ti:sapphire laser oscillator (Coherent, Mira) produces a train of femtosecond pulses, which generates linearly polarized broadband $\mathrm{THz}$ pulses in a photoconducting switch TeraSED (Giga-Optics). A gated detection scheme based on electrooptic sampling, with a $1 \mathrm{~mm}$ thick [110] ZnTe crystal as a sensor, allows us to measure the time profile of the electric field of the transmitted THz pulse (see Ref. 23 for further details). 
IR reflectivity and THz complex permittivity spectra were carefully fitted assuming the dielectric function in the form of generalized, factorized damped harmonic oscillator model ${ }^{24}$

$$
\varepsilon^{*}(\omega)=\varepsilon_{\infty} \prod_{j=1}^{n} \frac{\omega_{L O j}^{2}-\omega^{2}+i \omega \gamma_{L O j}}{\omega_{T O j}^{2}-\omega^{2}+i \omega \gamma_{T O j}}
$$

where $\omega_{\mathrm{TO}}$ and $\omega_{\mathrm{LO}}$ are the frequencies of $j$-th transverse optic (TO) and longitudinal optic (LO) phonons, and $\gamma_{\mathrm{TO} j}$ and $\gamma_{\mathrm{LO} j}$ are the corresponding damping constants. $\varepsilon_{\infty}$ is the high-frequency (electronic) contribution to the permittivity, determined from the room-temperature frequencyindependent reflectivity tail, above the phonon frequencies. The temperature dependence of $\varepsilon_{\infty}$ was neglected, consistent with its behavior in other related perovskite dielectrics; ${ }^{25} \varepsilon^{*}(\omega)$ is related to the reflectivity $R(\omega)$ of the bulk sample by

$$
R(\omega)=\left|\frac{\sqrt{\varepsilon^{*}(\omega)}-1}{\sqrt{\varepsilon^{*}(\omega)}+1}\right|^{2} .
$$

Raman scattering experiments were performed in the frequency range of $10-1600 \mathrm{~cm}^{-1}$ and the temperature range of 5-300 K. Unpolarized spectra excited with a $514.5 \mathrm{~nm}$ line of Ar laser were recorded in back-scattering geometry with a Renishaw RM 1000 micro-Raman spectrometer equipped with Bragg filters. Laser power of $\sim 10 \mathrm{~mW}$ was focused on a $\sim 5 \mu \mathrm{m}$ spot. The spectra were curve-fitted to a sum of damped harmonic oscillators, using in-house software.

\section{Theoretical Calculations}

Density functional theory (DFT) calculations were performed with the Vienna Ab-initio Simulation Package. PBEsol pseudopotentials with $\mathrm{Pb} 5 d$, 6s, and 6p; Mn 3s, 3p, 3d, and 4s; Te $5 s$ and $5 p$; and oxygen $2 s$ and $2 p$ valence states were used. A $500 \mathrm{eV}$ plane wave cutoff was used for all calculations. A $k$-point mesh with approximately $0.2 \AA^{-1}$ spacing between each point was used. Structural relaxations were considered converged once the force on each atom was less than $1 \mathrm{meV} / \AA$. The Dudarev approach to DFT $+U$ was used to approximate electronic correlation in the Mn $d$-orbitals. Reported results used $U=5 \mathrm{eV}$. Varying $U$ between 3 and $7 \mathrm{eV}$ did not significantly alter our results. The Isotropy Software Package was used to study symmetry related properties. 


\section{Results}

\section{Crystal Structure}

PXD of $\mathrm{Pb}_{2} \mathrm{MnTeO}_{6}$ demonstrates the formation of a pure perovskite-based compound (Figure 1). The crystal structure refinements were performed from PND at RT and $14 \mathrm{~K}$, and confirmed by SPXD (Supporting Information (SI), Figure S1). At $\mathrm{RT} \mathrm{Pb}_{2} \mathrm{MnTeO}_{6}$ is refined with the monoclinic space group $I 2 / m$ (No. 12). Figure 2a shows the excellent agreement between PND experimental and calculated data and the inset show how other space groups cannot explain the splitting of some of the reflections. Only $P 2_{1} / n$ could explain the data similar to $I 2 / m$, but the reflections $h+k+l \neq 2 n$ do not appear, which indicates $I$-centering of the unit cell. Moreover, the $R$ factor for the $I 2 / m$ model $\left(\mathrm{R}_{\mathrm{Bragg}}=3.83 \%\right)$ is significantly better than that of the $P 2_{1} / n$ model $\left(R_{\text {Bragg }}=5.72 \%\right)$. There are only a few other reported double perovskites with the same symmetry including $\mathrm{Pb}_{2} \mathrm{CoTeO}_{6}, \mathrm{~Pb}_{2} \mathrm{CoWO}_{6}, \mathrm{Sr}_{2} \mathrm{CoOsO}_{6}$ and $\mathrm{Sr}_{2} \mathrm{CoTeO}_{6}{ }^{26-29}$ The cell parameters of $I 2 / \mathrm{m}$ model are related to the ideal cubic perovskite aristotype $\left(a_{0} \approx 4 \AA\right)$ as $a \approx b \approx \sqrt{ } 2 a_{0}$, and $c \approx 2 a_{0}$. It is defined with one single A-site for Pb atoms at $4 i(x, 0, z)$, two crystallographically independent B positions for $\mathrm{Mn}$ at $2 a(0,0,0)$ and Te at $2 c(0,0,1 / 2)$ and two nonequivalent oxygen atoms (O1 at $4 i(x, 0, z)$ and $\mathrm{O} 2$ at $8 j(x, y, z))$. Table 1 illustrates the refined crystallographic parameters, atomic coordinates, thermal parameters and reliability factors at RT and Table 2 illustrates the interatomic distances and bond angles. At RT there is no significant movement of the $\mathrm{Pb}$ atom from the center of the polyhedron. The average $<\mathrm{Pb}-\mathrm{O}>$ bond distance at $\mathrm{RT}$ is $2.874 \AA$, comparable to that expected from the ionic radii sums of $2.89 \AA$ for ${ }^{\mathrm{XII}} \mathrm{Pb}^{2+}$ (i.r.: $1.49 \AA$ ) and ${ }^{\mathrm{VI}} \mathrm{O}^{2-}$ (i.r.: $1.40 \AA$ ); ${ }^{30}$ and also similar to $\mathrm{Pb}-\mathrm{O}$ distances in other double perovskites, e.g., $2.814 \AA$ in $\mathrm{Pb}_{2} \mathrm{MnReO}_{6}{ }^{31}$ and $2.898 \AA$ in $\mathrm{Pb}_{2} \mathrm{MnWO}_{6} \cdot{ }^{15}$ In addition, $\mathrm{MnO}_{6}$ and $\mathrm{TeO}_{6}$ are regular and do not demonstrate cationic displacements. The average $<\mathrm{Mn}-\mathrm{O}>$ and $<$ TeO> bond distances at RT, $2.159 \AA$ and $1.915 \AA$, respectively, are somewhat smaller than the calculated values for ${ }^{\mathrm{VI}} \mathrm{Mn}^{2+}$ (i.r.(High Spin): $0.83 \AA$ ) and $\mathrm{O}^{2-}$ of $2.23 \AA$ and ${ }^{\mathrm{VI}} \mathrm{Te}^{6+}$ (i.r.: $0.56 \AA$ ) and $\mathrm{O}^{2-}$ of $1.96 \AA \AA$, from Shannon ionic radii tables. ${ }^{30}$ However, similar Pb-based double perovskites, $\mathrm{Pb}_{2} \mathrm{MnWO}_{6}$ and $\mathrm{Pb}_{2} \mathrm{MnReO}_{6}$, have similar Mn-O bond distances (2.124 $\AA$ and 2.106(5) $\AA$, respectively), and $\mathrm{Pb}_{2} \mathrm{CoTeO}_{6}$ has a comparable Te-O distance (1.929(1) $\AA$ ) as well. ${ }^{26}$ demonstrated by DSC (SI, Figure S2), $C_{P}(T)$, dielectric measurements, IR and Raman spectroscopies (see 
below). The PND data at $14 \mathrm{~K}$ (Figure $2 \mathrm{~b}$ ) are explained with the monoclinic $C 2 / c$ (No.15) space group, with a relation of the unit cell parameters to those of the ideal cubic perovskite as $a \approx c \approx \sqrt{ } 6 a_{0}$, and $b \approx \sqrt{ } 2 a_{0}$. This low-temperature phase is unusual for perovskites. It was described for similar $\mathrm{Pb}_{2} \mathrm{RSbO}_{6}$ double perovskites ( $\mathrm{R}=$ rare earths $)^{32,33}$; which contain a completely ordered array of alternating $\mathrm{BO}_{6}$ and $\mathrm{B}^{\prime} \mathrm{O}_{6}$ octahedra sharing corners, tilted in antiphase along the three pseudocubic axes (with an a-b-b- tilting scheme). Also the transition sequence from $C 2 / c-$ to $-12 / \mathrm{m}$ space groups has been never reported before, since for $\mathrm{Pb}_{2} \mathrm{RSbO}_{6}$ the sequence is $C 2 / c \rightarrow P 2_{1} / n \rightarrow R 3 \rightarrow F m 3 m$, different from the one observed in $\mathrm{Pb}_{2} \mathrm{MnTeO}_{6}$. In $\mathrm{C2} / \mathrm{c}$ structure, $\mathrm{Pb}$ atoms are placed at the Wyckoff $8 f(x, y, z)$ A sites; $\mathrm{Mn}$ and Te atoms at the $4 e(0, y, 1 / 4)$ and $4 d(1 / 4,1 / 4,1 / 2) B$ and $B^{\prime}$ sites, respectively; and oxygen atoms are located at three crystallographically nonequivalent positions at $8 f(x, y, z)$. The Bragg $R$-factors obtained at $14 \mathrm{~K}$ for the refinement of the two PND frames are $5.82 \%$ and $6.32 \%$. The refinement of the occupancy of Pb and $\mathrm{O}$ atoms indicates full occupancies; no oxygen vacancies at any position and no antisite disorder between $\mathrm{Mn}$ and Te, at RT and $14 \mathrm{~K}$. Tables 1 and 2 also illustrate the refined parameters at $14 \mathrm{~K}$. This structure is confirmed by SPXD data collected at $11 \mathrm{~K}$ (see Figure S1a), showing that the magnetic diffraction peaks discussed below do not have any nuclear contribution from a lower lattice symmetry. SPXD (Figure S1b) also reveal the same structure at $50 \mathrm{~K}$, proving that the only structural transition is the one in the range $120 \mathrm{~K}$ to $160 \mathrm{~K}$; i.e., the $20 \mathrm{~K}$ transition is purely magnetic. Structural parameters from the $\mathrm{X}$-ray refinements are given in Table S1. The following analysis is based on the neutron data, which are much more accurate.

At low temperature the structure changes, significantly increasing the distortion. $\mathrm{Pb}$ atoms are now situated in distorted polyhedra, in which we can consider 8-fold coordination if we discard the $\mathrm{Pb}-\mathrm{O}$ distances longer than $3.1 \AA$. Pb atoms present off-center displacement, due to the $6 s^{2}$ lone pair. There are three $\mathrm{Pb}-\mathrm{O}$ shorter distances creating a $\mathrm{PbO}_{3} \mathrm{E}$ environment (E being the lone pair), as was described in $\mathrm{Pb}_{2} \mathrm{TmSbO}_{6}{ }^{33}$ The right inset of Figure $1 \mathrm{~b}$ shows the environment of $\mathrm{Pb}$ atoms and the arrow marks the direction of their displacement, which is almost along the $b$ direction. This antiparallel displacement of $\mathrm{Pb}$ from the centroids of their polyhedra motivated the study of possible antiferroelectric character in this compound. The Pb off-centering also implies the displacement of Mn cations inside their octahedra (0.044 $\AA$ ) closer to one octahedron edge formed by the O3 atoms (Table 1) and moving away from the Pb-O shorter distances (see left inset of Figure 2b); while Te cations are not displaced. This off-center displacement is highly unusual for the magnetic and JahnTeller-inactive $\mathrm{Mn}^{2+}\left(\mathrm{d}^{5}\right)$ cation, mimicking the behavior which usually occurs for $d^{0}$ cations.

The phenomenological Brown's Bond-Valence Model (BVS) ${ }^{34}$ helps to estimate the valences of the cations, by an empirical relationship between the observed bond-lengths and the valence of a bond. The values obtained at RT, Mn (2.22(1)+) and Te (6.04(3)+), are close to the expected values of 2+ and 6+; although the slightly higher valence observed for Mn could suggest a minor compressive stress on these bonds. We corroborate the oxidation states of $\mathrm{Mn}^{2+}$ and $\mathrm{Te}^{6+}$ by $\mathrm{X}$ ray absorption spectroscopy (SI, Figure S3). 


\section{Magnetic Measurements}

The magnetization vs. temperature curves recorded in ZFC and FC modes (Figure 3a) show that $\mathrm{Pb}_{2} \mathrm{MnTeO}_{6}$ undergoes an $\mathrm{AFM}$ transition at $T_{\mathrm{N}} \sim 20 \mathrm{~K}$, as observed by a drop in the magnetization. ZFC and FC curves show no divergence in the entire 5-300 K temperature region. The high temperature data were fitted to a Curie-Weiss law, $\chi=C /\left(T-\theta_{\mathrm{CW}}\right.$ ) (inset (i) of Figure 3a). The fitting allowed us to extract the effective magnetic moment, $\mu_{\text {eff }}=5.98 \mu_{\mathrm{B}}$, which agrees well with $\mu_{\mathrm{Mn} 2+}=5.92 \mu_{\mathrm{B}}$. The Curie-Weiss constant, $\theta_{\mathrm{CW}}=-105 \mathrm{~K}$, indicating strong antiferromagnetic correlations in the system. Note that the fit was done for the temperature range above the structural transition ( $\mathrm{T}>160 \mathrm{~K}$ ). In inset (ii) of Figure 3a the isothermal magnetization curves are shown. They indicate a linear paramagnetic behavior at $300 \mathrm{~K}$ and an AFM behavior at $5 \mathrm{~K}$ showing a lack of saturation even at the highest measured field (as expected for an AFM material); however a slight curvature is observed in the M vs $\mathrm{H}$ data at $5 \mathrm{~K}$, indicating small competition of magnetic interactions at low temperature.

\section{Magnetic Structure}

The magnetic structure determination was carried out from PND at $14 \mathrm{~K}$. New reflections of magnetic origin appear in the PND diagram (SI, Figure S4), which can be indexed with the $\boldsymbol{k}=$ ( $\left.\begin{array}{lll}0 & 1 & 0\end{array}\right)$ propagation vector. The magnetic symmetry analysis has been performed with the ISODISTORT software. ${ }^{35}$ The collinear AFM solution was found with a magnetic space group $P_{C} 2 / n$, subsequently transformed into the set of constraints to be used in the Fullprof program. ${ }^{22}$ After the full refinement of the profile, including the magnitude of the magnetic moments, the best discrepancy factor of $R=9.78 \%$ was obtained. The magnetic moment on the Mn atom has a value of $3.64(9) \mu_{\mathrm{B}}$ and is aligned along the $b$ axis of the $C 2 / c$ unit cell $(<110>$ axis of conventional perovskite subcell). A view of the magnetic structure is displayed in Figure 3b. The magnetic moments on $\mathrm{Mn}$ atoms are ferromagnetically arranged into chains running along the $b$ axis. Each chain is surrounded by four nearest neighbour chains with the opposite direction of spins, resulting in the AFM structure. The long distance superexchange magnetic interactions via Mn-O-Te-O-Mn paths, account for the relatively low $T_{\mathrm{N}}$. Other examples of AFM double 
perovskites with similar $T_{\mathrm{N}}$ and containing one magnetic cation at B site and one non-magnetic ion at $\mathrm{B}^{\prime}$ are $\mathrm{Sr}_{2} \mathrm{CrSbO}_{6}\left(T_{\mathrm{N}}=12 \mathrm{~K}\right),{ }^{36} \mathrm{Sr}_{2} \mathrm{FeWO}_{6}\left(T_{\mathrm{N}}=37 \mathrm{~K}\right)^{37}$ and $\mathrm{Pb}_{2} \mathrm{CoTeO}_{6}\left(T_{\mathrm{N}}=16 \mathrm{~K}\right){ }^{26}$

\section{Heat Capacity}

Figure 4 shows the temperature dependence of $C_{\mathrm{P}}$. The peaks seen near $117 \mathrm{~K}$ (cooling) and $160 \mathrm{~K}$ (heating) indicate a structural phase transition that exhibits a large thermal hysteresis, characteristic of a first-order phase transition. Similar values of critical temperature $\left(T_{\mathrm{C}}\right)$ were obtained with DSC (SI, Figure S2). The measurements were reproducible and the change of enthalpy was $0.45 \pm 0.01 \mathrm{~J} / \mathrm{g}$ at $T_{\mathrm{C}}$. The peak near $17.2 \mathrm{~K}$ manifests the AFM ordering, which shifts down by $0.4 \mathrm{~K}$ in external magnetic field of $9 \mathrm{~T}$ (Inset of Figure 4).

\section{Dielectric and ac Conductivity Measurements}

Dielectric data (Figure 5) are strongly influenced by leakage conductivity of the sample. Note that the conductivity $\sigma^{\prime}$ drastically increases with both frequency and temperature (Figure 5c); at $50 \mathrm{~K}$ the $\sigma$ is 6 orders of magnitude higher at $900 \mathrm{kHz}$ than at $1 \mathrm{~Hz}$. This is caused by a nonhomogeneous conductivity in the ceramic sample; the grains exhibit large conductivity (seen at high frequencies), while the grain boundaries are less conducting and therefore they radically reduce the low-frequency $\sigma$. The resistive grain boundaries are responsible for the creation of internal barrier layer capacitors with ultra low thickness. They enhance the electric capacity of the sample and cause a "giant" effective permittivity at low frequencies, which reaches in our case values about 10,000 at temperatures above $200 \mathrm{~K}$ (SI, Figure S5). This mechanism of creation of "giant" or "colossal" dielectric permittivity is well known in many dielectrics and multiferroics with non-negligible conductivity. ${ }^{38}$ The conductivity is also responsible for the high dielectric loss (Figure 5b). Nevertheless, the influence of conductivity on $\varepsilon^{\prime}$ decreases with increasing frequency, therefore $\varepsilon^{\prime}$ in $\mathrm{Pb}_{2} \mathrm{MnTeO}_{6}$ seen at $900 \mathrm{kHz}$ has intrinsic values, at least, up to $200 \mathrm{~K}$. In Figure 5a, one can see remarkable step-down of $\varepsilon^{\prime}(T)$ near $120 \mathrm{~K}$ (on cooling), typical for antiferroelectric ${ }^{39}$ or improper-ferroelectric ${ }^{40}$ phase transitions. According to our structural refinement, the low-temperature $C 2 / c$ crystal structure is centrosymmetric, thus the ferroelectric order is excluded. Similar change of $\varepsilon^{\prime}(T)$ is seen on heating, but the anomaly occurs 
$\sim 40 \mathrm{~K}$ higher, which is evidence for a first-order character of the phase transition. The temperatures of dielectric anomalies correspond well to the temperatures of the phase transitions seen in $C_{\mathrm{P}}(T)$ (Figure 4). The centrosymmetric character of the both phases was also confirmed by Second Harmonic Generation (SHG) that showed only very weak SHG response (close to the noise limit) even with an unusually long collection time.

Since the unit cell doubles below $T_{C}$ in $\mathrm{Pb}_{2} \mathrm{MnTeO}_{6}$ like in antiferroelectrics and the step down of $\varepsilon^{\prime}$ observed at $T_{\mathrm{C}}$ is reminiscent of a dielectric anomaly at antiferroelectric phase transition, we tried to measure antiferroelectric hysteresis loops. Only linear increase of polarization with electric field typical for paraelectrics was observed (see SI Figure S6), but we cannot exclude that antiferroelectric hysteresis loop appears at higher electric fields than our $28 \mathrm{kV} / \mathrm{cm}$, however, we could not apply higher field due to a finite conductivity of the sample.

Similar step down of permittivity as in our case has been observed in other double perovskites including $\mathrm{Pb}_{2} \mathrm{CoTeO}_{6}{ }^{26}$ and $\mathrm{Pb}_{2} \mathrm{MnWO}_{6}{ }^{15}$ Authors of both references claimed that their lowtemperature phases are antiferroelectric although they did not measure hysteresis loops. Here we would like to stress that both materials are likely antipolar and only hysteresis loop measurements can distinguish between antiferroelectric (switchable polarization) and antipolar (non-switchable) behavior.

\section{Infrared and THz Spectroscopy}

Intrinsic $\varepsilon^{\prime}$ is determined by the sum of phonon and electron contributions to the permittivity (it corresponds to the low-frequency edge of THz $\varepsilon^{\prime}$ in Figure $6 \mathrm{~b}$ and c), so if $\varepsilon^{\prime}(T)$ changes near $T_{\mathrm{C}}$ (120-150 K), some phonon shifts and splittings are expected. For that reason, IR reflectivity and $\mathrm{THz}$ transmission spectra were measured down to $12 \mathrm{~K}$. Dramatic changes of the reflectivity with temperature can be observed in Figure 6a. Four asymmetric reflection bands abruptly split below $T_{\mathrm{C}}$. Fits of IR reflectivity and experimental $\mathrm{THz}$ permittivity reveal $16 \mathrm{IR}$ active (i.e. polar) phonons above $150 \mathrm{~K}$ and 30 phonons at lower temperatures (see mode parameters in SI, Table S2). Jumps in phonon frequencies are clearly seen near $T_{\mathrm{C}}$ in the temperature dependence of polar phonon frequencies (SI, Figure S7). For the explanation of the number of observed phonons, we performed factor group analysis of lattice vibrations, using the known site symmetries of atoms, obtained from our structural investigations. In the high-temperature phase 
$I 2 / m$, the primitive unit cell contains one formula unit with 10 atoms with 30 degrees of freedom, and therefore, 30 phonons are expected. The following phonon counts and symmetries in the Brillouin Zone (BZ) center were obtained:

$$
\Gamma_{I 2 / m}=7 A_{g}\left(x^{2}, x y\right)+7 A_{u}(z)+5 B_{g}(x z, y z)+11 B_{1 u}(x, y)
$$

Here, $x, y$, and $z$ mark electric polarizations of the IR radiation for which the phonons are IR active, whereas the rest of the symbols are components of the Raman tensor. After subtraction of $1 A_{u}$ and $2 B_{1 u}$ symmetry acoustic phonons, 15 IR and 12 Raman active phonons are expected in the spectra of the high-temperature phase.

In the low-temperature phase the crystal structure changes to $C 2 / c$ and the unit cell doubles, so 60 phonons are expected. We obtained the following symmetries and activities of the phonons:

$$
\Gamma_{C 2 / c}=13 A_{g}\left(x^{2}, x y\right)+16 A_{u}(z)+14 B_{g}(x z, y z)+17 B_{u}(x, y)
$$

Likewise after subtraction of 3 acoustic modes, 30 phonons are IR active and 27 phonons should be active in the Raman spectra. As expected, 30 phonons were observed in the IR spectra below $130 \mathrm{~K}$ (Figure 6) and 15 polar phonons in the high-temperature $I 2 / m$ phase, which exactly correspond to the factor group analysis. One extra mode observed in both phases can be a geometrical resonance in anisotropic media, caused by two LO modes with similar frequencies. ${ }^{41}$ An alternative possible explanation is that the extra mode is very weak (one near $20 \mathrm{~cm}^{-1}$ - see SI, Figure S8), which can be a central mode, that describes dynamical hopping of Pb cations among several equivalent positions. This central mode is seen only in the THz dielectric loss and can be as well caused by multiphonon absorption. The only surprising observation is, that this very weak and heavily damped mode remains in the $\mathrm{THz}$ spectra even in the low-temperature phase, where both central mode and multiphonon absorption should disappear. The last and most probable explanation for the extra mode is that it is a defect-induced mode, which can be heavily damped, if its frequency lies in the range of acoustic phonons. ${ }^{42}$

\section{Raman Spectroscopy}

Phonons in the Raman scattering spectra (Figure 7) exhibit gradual decrease of damping on cooling and an abrupt change between 140 and $130 \mathrm{~K}$, where $\mathrm{Pb}_{2} \mathrm{MnTeO}_{6}$ undergoes the phase transition. Some of the modes shift and some of them split below $T_{\mathrm{C}}$ (see temperature 
dependence of mode frequencies in Figure 8). We can again compare the number of observed phonons with theoretically predicted phonons. In the high-temperature phase, we see 11 Raman active phonons instead of 12 allowed by symmetry. At low temperatures we observed 15 Raman active modes instead of the 27 allowed. The missing modes can be explained by their weak intensity, by their overlapping with other strong modes, or by contributions of multiple differently oriented grains to the spectra, if the grain size is smaller than the laser-spot size (about $2 \mu \mathrm{m}$ ). The grain size of $\mathrm{Pb}_{2} \mathrm{MnTeO}_{6}$ is around 130-200 nm. More detailed Raman scattering experiments performed on $\mathrm{Pb}_{2} \mathrm{MnTeO}_{6}$ single crystals are required to resolve all allowed Raman-active modes.

Both Raman and IR spectra support a first order structural phase transition. Most of the phonons exhibit abrupt shifts and splitting at $T_{\mathrm{C}}$. Changes of polar phonon frequencies and related changes of their dielectric strengths, due to Lyddane-Sachs-Teller relation, are responsible for the drop down of $\varepsilon^{\prime}(T)$ below $T_{\mathrm{C}}$. On the other hand, no soft mode activated from hightemperature BZ boundary due to multiplication of the unit cell was observed in the spectra below $T_{\mathrm{C}}$. The latter indicates that the structural, and highly probable, antipolar phase transition is not driven by a soft phonon from the BZ boundary. Such soft phonons were observed in the Raman spectra of $\mathrm{SrTiO}_{3}$ below its antiferrodistortive phase transition at $105 \mathrm{~K}^{43}$ Note as well that BZcenter phonon softening was observed near $T_{C}$ in both IR and Raman spectra ${ }^{39,44}$ of canonical antiferroelectric $\mathrm{PbZrO}_{3}$, although no phonon softening was observed at the BZ boundary in this material. $^{45}$

\section{Density Functional Theory Calculations}

$\mathrm{Pb}_{2} \mathrm{MnTeO}_{6}$ was relaxed in the expected high symmetry cubic Fm-3m structure. The computed cubic Fm-3m structure has a lattice constant for the conventional cell of $8.073 \AA$ and the Mn-O bond lengths are $0.172 \AA$ shorter than the Te-O bond lengths. We computed the phonons at the zone center and zone boundary points, showing that the cubic structure is unstable to polar distortions and to a variety of oxygen octahedron rotation patterns. $I 2 / \mathrm{m}$ symmetry is established by the addition of an $a^{-} a^{-} c^{0}$ octahedral rotation pattern to the cubic structure, and $C 2 / c$ symmetry by the addition of an $L_{3}^{+}$mode, which primarily corresponds to a complex pattern of oxygen 
octahedra rotations about the [001] cubic axis. The $L_{3}^{+}$mode also allows the Pb ions to offcenter along the $b$ axis in an antipolar ordering, accounting for most of the observed offcentering pattern. A small deviation of the $\mathrm{Pb}$ ions displacement from the $b$ direction is from GM5+ mode - see the lattice distortion in $C 2 / c$ structure in Figure 8 . The $I 2 / m$ and $C 2 / c$ structures were relaxed with a variety of spin orderings on the Mn ions. The lowest energy magnetic ordering for each structure is the AFM ordering observed experimentally (Figure 3b). The relaxed AFM $C 2 / c$ structure is our calculated ground state, in agreement with the experimental low temperature structure. The structural parameters of the relaxed $I 2 / m$ and $C 2 / c$ structures are presented in Table S3 (SI), showing excellent agreement with the structures of the phases experimentally observed at RT and $14 \mathrm{~K}$, respectively.

The presence of an antipolar ordering of $\mathrm{Pb}$ cation displacements in the $C 2 / c$ phase suggests the possibility of antiferroelectricity. To explore this, we searched for a low energy metastable polar structure that could be induced by an applied electric field. ${ }^{46}$ First, we relaxed the structures obtained by freezing in the unstable polar mode along various directions ([100], [110] and [111]) leading to structures with 10 atoms/primitive cell and space groups $I 4 m m$, Imm2, and $R 3 m$, respectively. As shown in Table 3, these structures are much higher in energy than the $C 2 / c$ ground state. Furthermore, none of these polar structures are local minima of the energy - each has multiple instabilities corresponding to various octahedral rotation patterns. In each case, the addition of octahedral rotation distortions and further relaxation yields a nonpolar structure, as detailed in Table 3, with the exception of $\operatorname{Imm} 2$ combined with $a^{0} a^{0} c^{+}$. In this case, the structure has $P m n 2_{1}$ symmetry, with a nonzero polarization along the [110] cubic direction, but is not a local minimum of the energy, exhibiting an instability corresponding to the $a^{-} a^{-} c^{0}$ rotation pattern. Relaxing this rotation pattern into the $P m n 2_{1}$ structure leads to the nonpolar $P 2_{1} / n$ structure. While a low-energy metastable polar phase with a more complex structure is not ruled out by our search, switching would require a change in the octahedral rotation pattern that would almost certainly result in a large switching barrier, and consequently a coercive field above the breakdown field. Therefore, we conclude that antiferroelectric switching is unlikely to be observed in $\mathrm{Pb}_{2} \mathrm{MnTeO}_{6}$.

\section{Conclusions}


We have succesfully prepared the novel double perovskite $\mathrm{Pb}_{2} \mathrm{MnTeO}_{6}$. This compound exhibits a first-order phase transition from a room temperature $I 2 / m$ structure to a low temperature (below $\sim 120 \mathrm{~K})$ C2/c structure. The number of phonons observed in the IR and Raman spectra correspond to the number of phonons expected from the factor group analysis in both phases. Abrupt changes at $T_{\mathrm{C}}$ in both IR and Raman spectra confirm first-order character of the phase transition seen as well in thermal capacity and dielectric permittivity measurements. The low temperature phase is characterized by large displacements of the $\mathrm{Pb}$ atoms, forming a $\mathrm{PbO}_{3} \mathrm{E}$ environment with shorter $\mathrm{Pb}-\mathrm{O}$ bonds. This distortion induces off-centering of $\mathrm{Mn}^{2+}$ magnetic cations from the center of the octahedra. Due to this strong antiparallel displacement the possible antiferroelectric character of the compound was investigated, but no antiferroelectric loops were detected, which suggests that the structure is only antipolar. First principles calculation confirms that antiferroelectric switching is unlikely to be observed in $\mathrm{Pb}_{2} \mathrm{MnTeO}_{6}$. Near $20 \mathrm{~K}$, magnetic, heat capacity and PND studies revealed an antiferromagnetic phase transition with a collinear antiferromagnetic structure at low temperatures. Thus, $\mathrm{Pb}_{2} \mathrm{MnTeO}_{6}$ belongs to a rare group of antipolar antiferromagnets with a potential large magnetoelectric coupling.

\section{Acknowledgments}

This work was supported by the NSF-DMR-0966829, the ARO-DOD-VV911NF-12-1-0172 grants. Thanks to Felix O. Saouma and Joon I. Jang for the Second Harmonic Generation Measurements. Use of the Spallation Neutron Source is supported by the Division of Scientific User Facilities, Office of Basic Energy Sciences, US Department of Energy, under contract DEAC05-00OR22725 with UT-Battelle, LLC. Use of the National Synchrotron Light Source, Brookhaven National Laboratory was supported by the DOE BES (DE-AC02-98CH10886). A.M.A. is grateful to the Russian Science Foundation for the financial support (grant 14-1300680). B.M.A. and C. J. F. were supported by the Army Research Office under grant No. W911NF-10-1-0345. The work of K. M. R. was supported by the Office of Naval Research N00014-12-1-1040. The work in Prague (JP, MS, DN, PV and SK) was supported by the Czech

Science Foundation Project No. 15-08389S. S.S. was supported by EU funding under the $7^{\text {th }}$ Framework Programme (Project NOTEDEV). Heat capacity studies in external magnetic field 
were performed in MLTL (see: http://mltl.eu), which is supported within the program of Czech Research Infrastructures (project No. LM2011025).

\section{Author Information}

Corresponding autor: martha@rutgers.edu

†Current address: Grupo de Energía y Química Sostenibles (EQS), Instituto de Catalisis y Petroleoquímica, CSIC, C/ Marie Curie, 2, L10, 28049 Madrid, Spain

† Current address: Department of Engineering Sciences, Uppsala University, Box 534, SE-75121 Uppsala, Sweden. 


\section{References}

(1) Kobayashi, K. I.: Kimura, T.; Sawada, H.: Terakura K.; Tokura, Y. Nature, 1998, 395, 677-680.

(2) Setter N.; Cross, L. E. J. Mater. Sci., 1980, 15, 2478-2482.

(3) Isupov V. A.; Krainik, N. N. Phys. Solid State, 1965, 6, 2975-2976.

(4) O’Dell, T. H. The Electrodynamics of Magneto-Electric Media (North-Holland, Amsterdam, 1970).

(5) Wood, V. E.; Austin, A. E. Int. J. Magn. 1974, 5, 303-315.

(6) Tokura, Y.; Seki, S.; Nagaosa, N. Rep. Prog. Phys. 2014, 77, 076501.

(7) Wang, J. ; Neaton, J. B. ; Zheng, H. ; Nagarajan, V. ; Ogale, S. B. ; Liu, B. ; Viehland, D. ; Vaithyanathan, V. ; (Schlom, D. G. ; Waghmare, U. V. ; Spaldin, N. A. ; Rabe, K. M. ; Wuttig M. ; Ramesh, R. Science 2003, 299, 1719-1722.

(8) Azuma, M.; Takata, K.; Saito, T.; Ishiwata, S.; Shimakawa, Y.; Takano, M. J. Am. Chem. Soc. 2005, 127, 88898892.

(9) Seshadri, R.; Hill, N. A. Chem. Mater. 2001, 13, 2892-2899.

(10) Kimura, T.; Goto, T.; Shintani, H.; Ishizaka, K.; Arima T.; Tokura, Y. Nature 2003, 426, 55-58.

(11) Barone P.; Picozzi, S. Comptes Rendus Physique 2015, 16, 143-152

(12) Singh K.; Simon, C.; Cannuccia, E.; Lepetit, M. B.; Corraze, B.; Janod, E.; Cario, L. Phys. Rev. Lett. 2014, 113, 137602

(13) Walsh, A.; Payne, D. J.; Egdell, R. G.; Watson, G. W. Chem. Soc.Rev. 2011, 40, 4455-4463.

(14) Larrégola, S. A.; Alonso, J. A.; Pedregosa, J. C.; Martínez-Lope, M. J.; Algueró, M.; De la Peña-O'shea, V.; Porcher, F.; Illas, F. Dalton Trans. 2009, 28, 5453-5459.

(15) Blasco, J.; Merino, R. I.; Garcia J.; Sanchez, M. C. J. Phys.: Condens. Matter, 2006, 18, 2261-2271.

(16) Jeong, I.-K.; Ahn, J. S.; Kim, B. G.; Yoon, S.; Singh, S. P.; Pandey, D. Phys. Rev. B 2011, 83, 064108.

(17) Martinez, R.; Palai, R.; Huhtinen, H.; Liu, J.; Scott, J. F.; Katiyar, R. S. Phys. Rev. B 2010, 82, 134104.

(18) R. Blinc, M. Kosec, J. Holc, Z. Trontelj, Z. Jaglicic, and N. Dalal, Ferroelectrics 2007, 349, 16-20.

(19) Evans, D. ; Schilling, A. ; Kumar, A. ; Sanchez, D. ; Ortega, N. ; Arredondo, M. ; Katiyar, R. ; Gregg, J. ; Scott, J. Nat. Commun. 2013, 4, 1534.

(20) Huq, A.; Hodges, J. P.; Gourdon, O.; Heroux, L. Z. Kristallogr. Proc. 2011, 1, 127-135.

(21) (a) Rietveld, H. M. J. Appl. Crystallogr. 1969, 2, $65-71$ (b) Rodríguez-Carvajal, J. Physica B 1993, 192, 55-69.

(22) A. Coelho. Topas-Academic v. 5 Technical Reference, 2013. Topas-Academic is available at www.topasacademic.net

(23) Kužel, P.; Němec, H.; Kadlec, F.; Kadlec, C. Opt. Express 2010, 18, 15338-15348.

(24) Gervais, F. Infrared and Millimeter Waves, edited by K. J. Button, Academic, New York 1983, vol 8, Chap. 7, 279.

(25) Rössle, M.; Wang, C. N.; Marsik, P.; Yazdi-Rizi, M.; Kim, K.W.; Dubroka, A.; Marozau, I.; Schneider, C. W.; Humlíček, J.; Baeriswyl D.; Bernhard C. Phys. Rev. B 2013, 88, 104110.

(26) Ivanov, S. A.; Nordblad, P.; Mathieu, R.; Tellgren R.; Ritter, C. Dalton Trans. 2010, 39, 11136-11148.

(27) Baldinozzi, G.; Calvarin, G.; Sciau, P.; Grebille D.; Suard, E. Acta Crystallogr. B, 2000, 56, 570-6. 
(28) Morrow, R.; Mishra, R.; Restrepo, O. D.; Ball, M. R.; Windl, W.; Wurmehl, S.; Stockert, U.; Büchner, B.; Woodward, P. M. J. Am. Chem. Soc. 2013, 135, 18824-18830.

(29) Ortega-San Martin, L.; Chapman, J. P.; Lezama, L.; Sanchez-Marcos, J.; Rodriguez-Fernandez, J.; Arriortuab, M. I.; Rojo T. J. Mater. Chem. 2005,15, 183-193.

(30) Shannon, R. D. Acta Crystallogr. A 1976, 32, 751-767.

(31) Blasco, J.; Lafuerza, S.; Garcia, J.; Subias, G.; Sanchez, M. C.; Cuartero, V.; Stankiewicz J. Dalton Trans. 2011, 40, 3211-3218.

(32) Larrégola, S. A.; Alonso, J. A.; De la Peña-O’Shea, V. A.; Sheptyakov, D.; Pomjakushin, V.; Fernandez-Díaz, M. T.; Pedregosa, J. C. Inorg. Chem. 2014, 53, 5609-5618.

(33) Larregola, S. A.; Alonso, J. A.; Sheptyakov, D.; Alguero, M.; Muñoz, A.; Pomjakushin, V.; Pedregosa J. C. J. Am. Chem. Soc. 2010, 132, 14470-14480.

(34) (a) Brese, N .E.; O’Keefe, M. Acta Crystallogr. B 1991, 47, 192-197. (b) Brown, I. D.; Dabkowski, A.; McCleary, A. Acta Crystallogr. B 1997, 53, 750-761.

(35) Campbell, B. J.; Stokes, H. T.; Tanner, D. E.; Hatch, D. M. J. Appl. Cryst. 2006, 39, 607-614.

(36) Retuerto, M.; García-Hernández, M.; Martínez-Lope, M. J.; Fernández-Díaz, M. T.; Attfield J. P.; Alonso, J. A. J. Mater. Chem. 2007, 17, 3555-3561.

(37) Azad, A. K.; Eriksson, S.-G.; Mellergard, A.; Ivanov, S. A.; Eriksen. J.; Rundlöf, H. Mater. Res. Bull. 2002, 37, 1797-1813.

(38) Lunkenheimer, P.; Krohns, S.; Riegg, S.; Ebbinghaus, S. G.; Reller, A.; Loidl A., Eur. Phys. J. Special Topics 2010, 180, 61-89.

(39) Ostapchuk, T.; Petzelt, J.; Železný, V.; Kamba, S.; Bovtun, V.; Porokhonskyy, V.; Pashkin, A.; Kužel, P.; Glinchuk, M. D.; Bykov, I. P.; Gorshunov, B.; Dressel, M. J. Phys.: Condens. Matter. 2001, 13, 2677-2689.

(40) Dvořák, V. Ferroelectrics 1974, 7, 1-9.

(41) Hlinka, J.; Ostapchuk, T.; Noujni, D.; Kamba, S.; Petzelt, J. Phys. Rev. Lett. 2006, 96, 027601.

(42) Barker A. S.; Sievers, A. J. Rev. Mod. Phys. 1975, 47, S1-S179.

(43) Fleury, P.A.; Scott, J. F.; Worlock, J. M. Phys. Rev. Lett. 1968, 21, 16.

(44) Hlinka, J.; Ostapchuk, T.; Buixaderas, E.; Kadlec, C.; Kužel, P.; Gregora, I.; Kroupa, J.; Savinov, M.; Klíč, A.; Drahokoupil, J.; Etxebarria, I.; Dec, J. Phys. Rev. Lett. 2014, 112, 197601.

(45) Tagantsev, A. K.; Vaideeswaran, K.; Vakhrushev, S. B.; Filimonov, A. V.; Burkovsky, R. G.; Shaganov, A.; Andronikova, D.; Rudskoy, A. I.; Baron, A. Q. R.; Uchiyama, H.; Chernyshov, D.; Bosak, A.; Ujma, Z.; Roleder, K.; Majchrowski, A.; Ko, J.-H.; Setter, N. Nat. Commun. 2013, 4, 2229.

(46) Rabe, K. Antiferroelectricity in Oxides: A Reexamination, in Functional Metal Oxides: New Science and Applications, eds. S. Ogale and V. Venkateshan Wiley-VCH Verlag GmbG \& Co. KGaA 2013. 


\section{Tables}

Table 1: Atomic parameters and agreement factors after the Rietveld refinement using PND data for $\mathrm{Pb}_{2} \mathrm{MnTeO}_{6}$ at RT and $14 \mathrm{~K}$.

\begin{tabular}{|c|c|c|c|}
\hline Temperature & $\mathrm{RT}$ & & $14 \mathrm{~K}$ \\
\hline$I 2 / m$ & & $C 2 / c$ & \\
\hline$a / \AA$ & $5.76346(7)$ & $a / \AA$ & $9.89484(18)$ \\
\hline$b / \AA$ & $5.71910(8)$ & $b / \AA$ & $5.68523(10)$ \\
\hline$c / \AA$ & $8.08374(10)$ & $c / \AA$ & $9.92111(18)$ \\
\hline$\beta / 0$ & $89.9310(15)$ & $\beta /{ }^{\circ}$ & $108.4296(13)$ \\
\hline$V / \AA^{3}$ & $266.455(6)$ & $V / \AA^{3}$ & $529.487(17)$ \\
\hline $\mathrm{Pb} 4 \mathrm{i}(\mathrm{x} 0 \mathrm{z})$ & & $\mathrm{Pb} 8 f(x y z)$ & \\
\hline$x$ & $0.5006(3)$ & $x$ & $0.6282(2)$ \\
\hline$z$ & $0.2484(3)$ & $y$ & $0.2699(4)$ \\
\hline$B / \AA^{2}$ & $1.62(1)$ & Z & $0.6257(2)$ \\
\hline $\operatorname{Mn} 2 a\left(\begin{array}{lll}0 & 0 & 0\end{array}\right)$ & & $B / \AA^{2}$ & $0.21(3)$ \\
\hline $\mathrm{B} / \AA^{2}$ & $0.67(4)$ & $\operatorname{Mn} 4 e\left(0 y^{1 / 4}\right)$ & \\
\hline Te $2 c\left(\begin{array}{lll}0 & 0 & 1 / 2\end{array}\right)$ & & $y$ & $0.7443(15)$ \\
\hline$B / \AA^{2}$ & $0.53(3)$ & $B / \AA^{2}$ & $0.09(12)$ \\
\hline 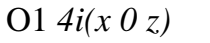 & & Te $4 d(1 / 41 / 41 / 2)$ & \\
\hline$x$ & $0.0430(5)$ & $B / \AA^{2}$ & $0.09(8)$ \\
\hline$z$ & $0.2647(3)$ & O1 8f(xyz) & \\
\hline$B_{11} / \AA^{2}$ & $0.0118(6)$ & $x$ & $0.8953(3)$ \\
\hline$B_{22} / \AA^{2}$ & $0.076(14)$ & $y$ & $0.2186(8)$ \\
\hline$B_{33} / \AA^{2}$ & $0.016(2)$ & $z$ & $0.9096(4)$ \\
\hline $\mathrm{O} 28 j(x y z)$ & & $B / \AA^{2}$ & $0.35(8)$ \\
\hline$x$ & $0.2667(3)$ & & \\
\hline$y$ & $0.2626(4)$ & $\mathrm{O} 28 f(x y z)$ & $0.1452(4)$ \\
\hline$z$ & $-0.0203(2)$ & $y$ & $0.0328(7)$ \\
\hline$B_{11} / \AA^{2}$ & $0.0115(5)$ & Z & $0.3571(5)$ \\
\hline$B_{22} / \AA^{2}$ & $0.0193(7)$ & $B / \AA^{2}$ & $0.54(8)$ \\
\hline$B_{33} / \AA^{2}$ & $0.0097(3)$ & O3 $8 f(x y z)$ & \\
\hline$B_{12} / \AA^{2}$ & $-0.0108(4)$ & $x$ & $0.6526(4)$ \\
\hline$B_{13} / \AA^{2}$ & $0.0025(4)$ & $y$ & $-0.0113(8)$ \\
\hline$B_{23} / \AA^{2}$ & $-0.0019(5)$ & $z$ & $0.8841(4)$ \\
\hline$\chi^{2}$ & 5.11 & $B / \AA^{2}$ & $0.54(7)$ \\
\hline$R_{\mathrm{p}}(\%)$ & 4.24 & & \\
\hline$R_{\mathrm{wp}}(\%)$ & 3.95 & $\chi^{2}$ (Pattern 1) & 3.65 \\
\hline$R_{\mathrm{I}}(\%)$ & 1.75 & $\chi^{2}$ (Pattern 2) & 2.36 \\
\hline \multirow[t]{9}{*}{$R_{\text {BraggCryst }}$} & 3.98 & $R_{\mathrm{p}}(\%)$ (Pattern 1) & 8.70 \\
\hline & & $R_{\mathrm{p}}(\%)$ (Pattern 2) & 13.1 \\
\hline & & $R_{\mathrm{wp}}(\%)($ Pattern 1$)$ & 8.71 \\
\hline & & $R_{\mathrm{wp}}(\%)$ (Pattern 2) & 11.5 \\
\hline & & $R_{\mathrm{I}}(\%)$ (Pattern 1) & 4.56 \\
\hline & & $R_{\mathrm{I}}(\%)$ (Pattern 2) & 7.51 \\
\hline & & $R_{\text {BraggCryst }}$ (Pattern 1) & 5.82 \\
\hline & & $R_{\text {BraggCryst }}$ (Pattern 2) & 6.32 \\
\hline & & $R_{\mathrm{BragaMa \sigma}}($ Pattern 2) & 9.78 \\
\hline
\end{tabular}


Table 2: Main interatomic distances $(\AA)$ and angles $\left(^{\circ}\right)$ for for $\mathrm{Pb}_{2} \mathrm{MnTeO}_{6}$ at $\mathrm{RT}$ and $14 \mathrm{~K}$.

\begin{tabular}{|c|c|c|c|}
\hline & RT & & $14 \mathrm{~K}$ \\
\hline $\mathrm{PbO}_{12}$ Polyhedra & & $\mathrm{PbO}_{12}$ Polyhedra & \\
\hline $\mathrm{Pb}-\mathrm{O}_{1}$ & $2.649(3)$ & $\mathrm{Pb}-\mathrm{O}_{1}$ & $3.209(4)$ \\
\hline $\mathrm{Pb}-\mathrm{O}_{1}$ & $3.125(3)$ & $\mathrm{Pb}-\mathrm{O}_{1}$ & $3.157(5)$ \\
\hline $\mathrm{Pb}-\mathrm{O}_{1}$ & $2.9770(3)$ & $\mathrm{Pb}-\mathrm{O}_{1}$ & $2.573(5)$ \\
\hline $\mathrm{Pb}-\mathrm{O}_{1}$ & $2.7674(3)$ & $\mathrm{Pb}-\mathrm{O}_{1}$ & $2.605(4)$ \\
\hline $\mathrm{Pb}-\mathrm{O}_{2}$ & $2.916(3)$ & $\mathrm{Pb}-\mathrm{O}_{2}$ & $2.790(5)$ \\
\hline $\mathrm{Pb}-\mathrm{O}_{2}$ & $2.666(3)$ & $\mathrm{Pb}-\mathrm{O}_{2}$ & $3.107(6)$ \\
\hline $\mathrm{Pb}-\mathrm{O}_{2}$ & $2.782(3)$ & $\mathrm{Pb}-\mathrm{O}_{2}$ & $2.982(5)$ \\
\hline $\mathrm{Pb}-\mathrm{O}_{2}$ & $3.022(3)$ & $\mathrm{Pb}-\mathrm{O}_{2}$ & $2.510(6)$ \\
\hline $\mathrm{Pb}-\mathrm{O}_{2}$ & $2.827(3)$ & $\mathrm{Pb}-\mathrm{O}_{3}$ & $2.967(5)$ \\
\hline $\mathrm{Pb}-\mathrm{O}_{2}$ & $3.063(3)$ & $\mathrm{Pb}-\mathrm{O}_{3}$ & $3.182(5)$ \\
\hline $\mathrm{Pb}-\mathrm{O}_{2}$ & $2.968(3)$ & $\mathrm{Pb}-\mathrm{O}_{3}$ & $2.886(5)$ \\
\hline $\mathrm{Pb}-\mathrm{O}_{2}$ & $2.724(3)$ & $\mathrm{Pb}-\mathrm{O}_{3}$ & $2.528(5)$ \\
\hline$<\mathrm{Pb}-\mathrm{O}>$ & $2.8739(9)$ & $<\mathrm{Pb}-\mathrm{O}>$ & $2.8746(9)$ \\
\hline $\mathrm{MnO}_{6}$ Octahedra & & $\mathrm{MnO}_{6}$ Octahedra & \\
\hline $\mathrm{Mn}-\mathrm{O}_{1}(\times 2)$ & $2.158(2)$ & $\mathrm{Mn}-\mathrm{O}_{1}(\times 2)$ & $2.159(5)$ \\
\hline $\mathrm{Mn}-\mathrm{O}_{2}(\times 4)$ & $2.158(2)$ & $\mathrm{Mn}-\mathrm{O}_{2}(\times 2)$ & $2.217(8)$ \\
\hline \multirow[t]{2}{*}{$<\mathrm{Mn}-\mathrm{O}>$} & $2.1585(9)$ & $\mathrm{Mn}-\mathrm{O}_{3}(\times 2)$ & $2.130(7)$ \\
\hline & & $<\mathrm{Mn}-\mathrm{O}>$ & $2.169(3)$ \\
\hline $\mathrm{TeO}_{6}$ Octahedra & & $\mathrm{TeO}_{6}$ Octahedra & \\
\hline $\mathrm{Te}-\mathrm{O}_{1}(\times 2)$ & $1.912(2)$ & $\mathrm{Te}-\mathrm{O}_{1}(\times 2)$ & $1.929(5)$ \\
\hline $\mathrm{Te}-\mathrm{O}_{2}(\times 4)$ & $1.916(2)$ & $\mathrm{Te}-\mathrm{O}_{2}(\times 2)$ & $1.920(4)$ \\
\hline \multirow[t]{2}{*}{$<\mathrm{Te}-\mathrm{O}>$} & $1.9146(9)$ & $\mathrm{Te}-\mathrm{O}_{3}(\times 2)$ & $1.938(4)$ \\
\hline & & $<\mathrm{Te}-\mathrm{O}>$ & $1.929(2)$ \\
\hline Angles around $\mathrm{O}$ & & Angles around $\mathrm{O}$ & \\
\hline $\mathrm{Mn}-\mathrm{O}_{1}-\mathrm{Te}$ & 166.32(10) & $\mathrm{Mn}-\mathrm{O}_{1}-\mathrm{Te}$ & $158.9(2)$ \\
\hline $\mathrm{Mn}-\mathrm{O}_{2}-\mathrm{Te}$ & $170.33(8)$ & $\mathrm{Mn}-\mathrm{O}_{2}-\mathrm{Te}$ & $161.2(4)$ \\
\hline \multirow[t]{2}{*}{$<\mathrm{Mn}-\mathrm{O}-\mathrm{Te}>$} & 168.32 & $\mathrm{Mn}-\mathrm{O}_{3}-\mathrm{Te}$ & $165.6(4)$ \\
\hline & & $<\mathrm{Mn}-\mathrm{O}-\mathrm{Te}>$ & 161.9 \\
\hline Bond Valence Mn & $2.22(1)$ & Bond ValenceMn & $2.16(2)$ \\
\hline Bond Valence Te & $6.04(3)$ & Bond Valence Te & $5.81(3)$ \\
\hline
\end{tabular}

Table 3: Calculated energies (density functional theory) of various combinations of polar distortions and octahedral rotations in meV/f.u. with respect to $F m-3 m$. The $C 2 / c$ ground state is included for energetic comparison. Ferromagnetic ordering of spins was assumed; for $C 2 / c$ this is $23 \mathrm{meV} / \mathrm{f}$.u. higher in energy than its ground state magnetic ordering.

\begin{tabular}{|c|c|c|c|c|}
\hline $\begin{array}{l}\text { Initial polar } \\
\text { distortion }\end{array}$ & $\begin{array}{l}\text { Additional } \\
\text { rotation } \\
\text { distortion }\end{array}$ & $\begin{array}{l}\text { Relaxed } \\
\text { distortion }\end{array}$ & $\begin{array}{l}\text { Relaxed } \\
\text { structure }\end{array}$ & $\begin{array}{l}\text { Energy } \\
\text { (meV/f.u.) }\end{array}$ \\
\hline - & - & - & $F m-3 m$ & 0 \\
\hline [100] & - & [100] & $\mathrm{I} 4 \mathrm{~mm}$ & -89 \\
\hline [110] & - & [110] & Imm2 & -96 \\
\hline [111] & - & [111] & $R 3 m$ & -100 \\
\hline [001] & $a^{0} a^{0} c^{+}$ & $a^{0} a^{0} c^{+}$ & $P 4 / m n c$ & -130 \\
\hline [110] & $a^{0} a^{0} c^{+}$ & {$[110]+a^{0} a^{0} c^{+}$} & $P m n 2_{1}$ & -145 \\
\hline [110] or [001] & $a^{0} a^{0} c^{-}$ & $a^{0} a^{0} c^{-}$ & $I 4 / m$ & -166 \\
\hline [110] or [001] & $a^{-} a^{-} c^{0}$ & $a^{-} a^{-} c^{0}$ & $I 2 / m$ & -213 \\
\hline [111] & $a^{-} a^{-} a^{-}$or $a^{-} a^{-} c^{0}$ & $a^{-} a^{-} a^{-}$ & $R-3$ & -213 \\
\hline [110] & $a^{-a} a^{-} c^{+}$ & $a^{-a} a^{-} c^{+}$ & $P 2_{1} / n$ & -216 \\
\hline- & $a a^{-} c^{0}+L_{3}^{+}$ & $a a^{-} c^{0}+L_{3}^{+}$ & $C 2 / c$ & -230 \\
\hline
\end{tabular}




\section{Figure Captions}

Figure 1. $\mathrm{PXD}$ pattern of $\mathrm{Pb}_{2} \mathrm{MnTeO}_{6}$ taken at room temperature (RT), with the structure refined in a double perovskite cell.

Figure 2. (a) Observed (crosses), calculated (full line) and difference (bottom) PND Rietveld profiles at RT. Upper inset shows the refinement for $\mathrm{Pb}_{2} \mathrm{MnTeO}_{6}$ in $R-3, I 4 / m$ and $I 2 / m$ space groups. (b) PND Rietveld profiles at $14 \mathrm{~K}$. The two sets of reflection patterns correspond to crystallographic and magnetic structures. Inset left: $\mathrm{TeO}_{6}$ (blue) and $\mathrm{MnO}_{6}$ (grey), where it is shown the displacement of $\mathrm{Mn}$ inside the Oh. Inset right: Environment of $\mathrm{Pb}$ cations and its offcenter.

Figure 3. (a) Susceptibility vs. temperature of $\mathrm{Pb}_{2} \mathrm{MnTeO}_{6}$ ceramics measured at $0.1 \mathrm{~T}$. Insets: (i) Inverse of the susceptibility fitted to the Curie Weiss Law. (ii) Hysteresis loops at $5 \mathrm{~K}$ and 300 K. (b) Magnetic structure with the spins over the Mn atoms (purple atoms). Te atoms are grey, $\mathrm{Pb}$ atoms yellow and oxygen red.

Figure 4. Temperature and magnetic field dependence of heat capacity. Large thermal hysteresis of $T_{C}$ is seen. $T_{N}$ exhibits $0.4 \mathrm{~K}$ shift down with an applied magnetic field.

Figure 5. Temperature dependence of (a) dielectric permittivity $\varepsilon$ ', (b) dielectric loss tan $\delta$ and (c) conductivity $\sigma$ ' measured at various frequencies on cooling (solid lines) and heating (dashed lines). Large thermal hysteresis of drop-down in $\varepsilon$ ' is typical for first-order phase transition.

Figure 6. (a) IR reflectivity spectra at selected temperatures together with (b) real and (c) imaginary part of complex dielectric permittivity obtained from fits of IR reflectivity. Symbols at low frequencies are experimental data obtained from time-domain $\mathrm{THz}$ spectrometer.

Figure 7: Raman scattering spectra taken at various temperatures. Abrupt change typical for structural phase transition of the first order is seen between 130 and $140 \mathrm{~K}$.

Figure 8: Temperature dependence of frequencies of Raman active phonons obtained from the fit of Raman spectra.

Figure 9: Antipolar $C 2 / c$ structure from DFT calculations. $\mathrm{Pb}$ and oxygen atoms are shown in black and red respectively. $\mathrm{MnO}_{6}$ and $\mathrm{TeO}_{6}$ octahedra are shaded in purple and brown respectively. $\mathrm{Pb}$ atoms are shown at their high symmetry positions with arrows indicating the direction of their displacements in C2/c. (a) View down the [110] cubic direction. The octahedral rotations in the $C 2 / c$ structure are clearly seen. (b) View down the [001] cubic direction. The 
complex rotation pattern about the [001] axis is evidenced. The motion of $\mathrm{Pb}$ along the $b$ axis is set by the $L_{3}^{+}$mode. The remainder of Pb ion motion is set by the GM5+ mode. 


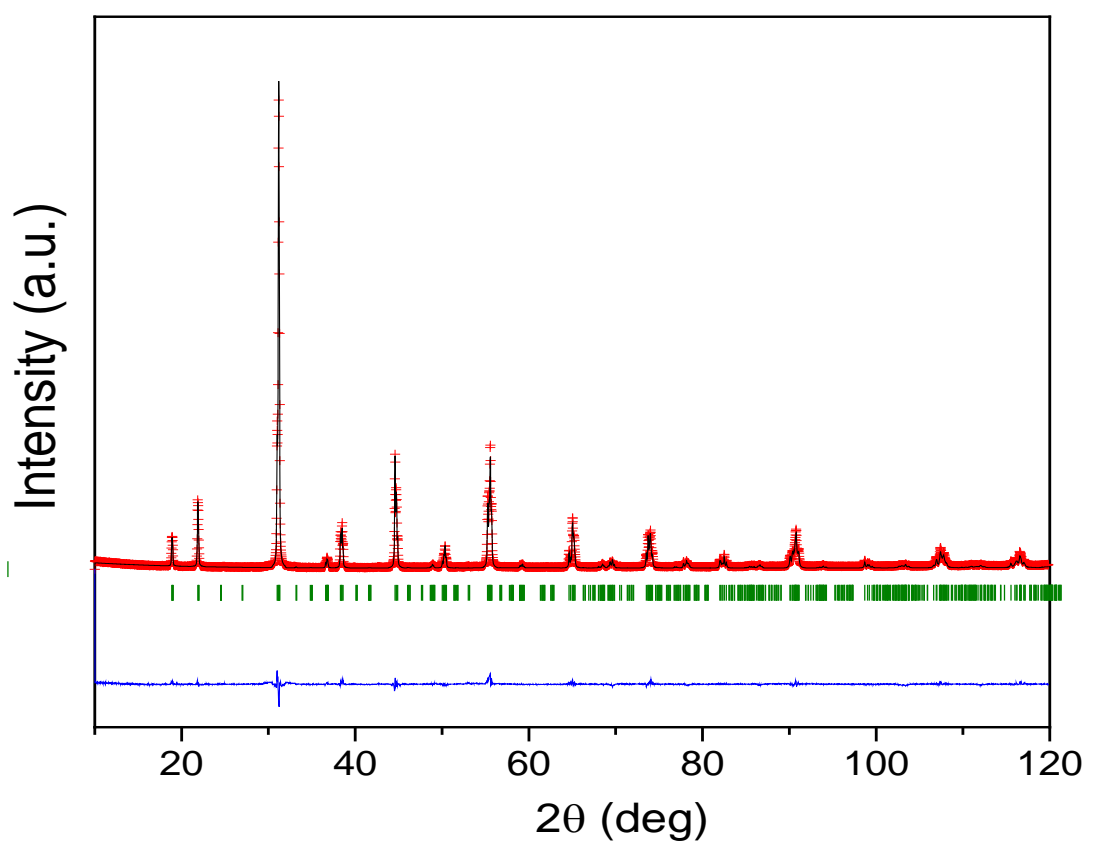

Figure 1 

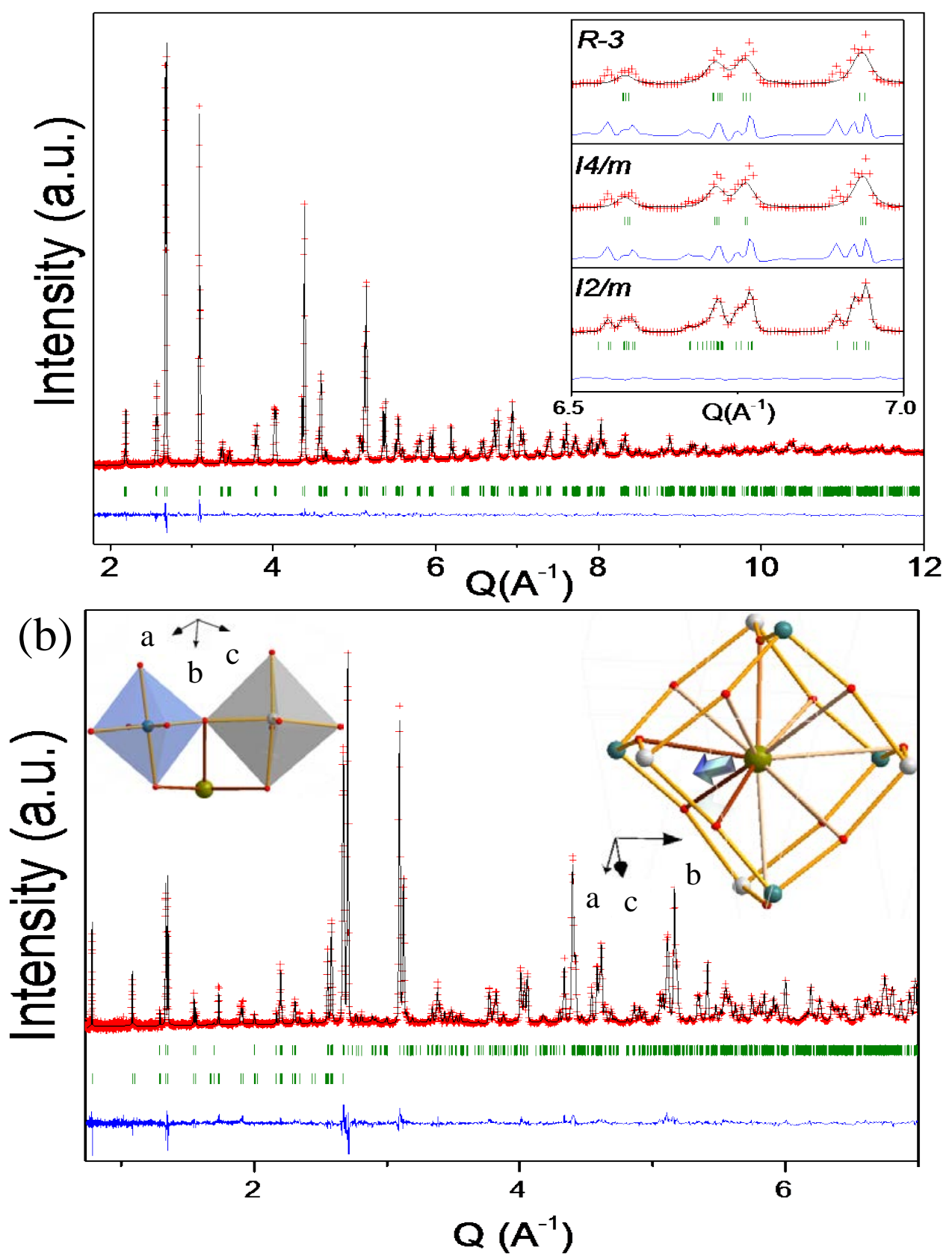

Figure 2 


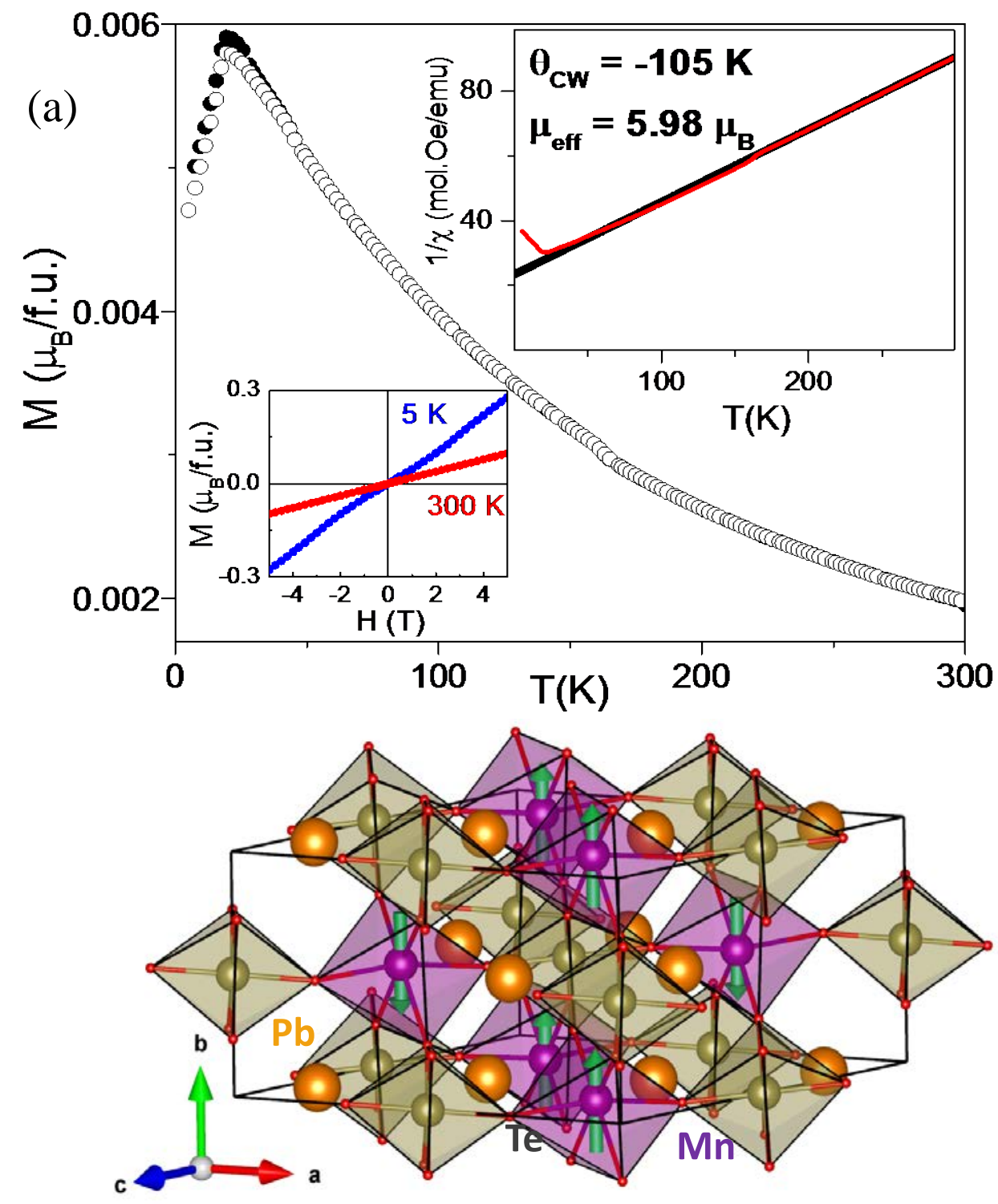

Figure 3 


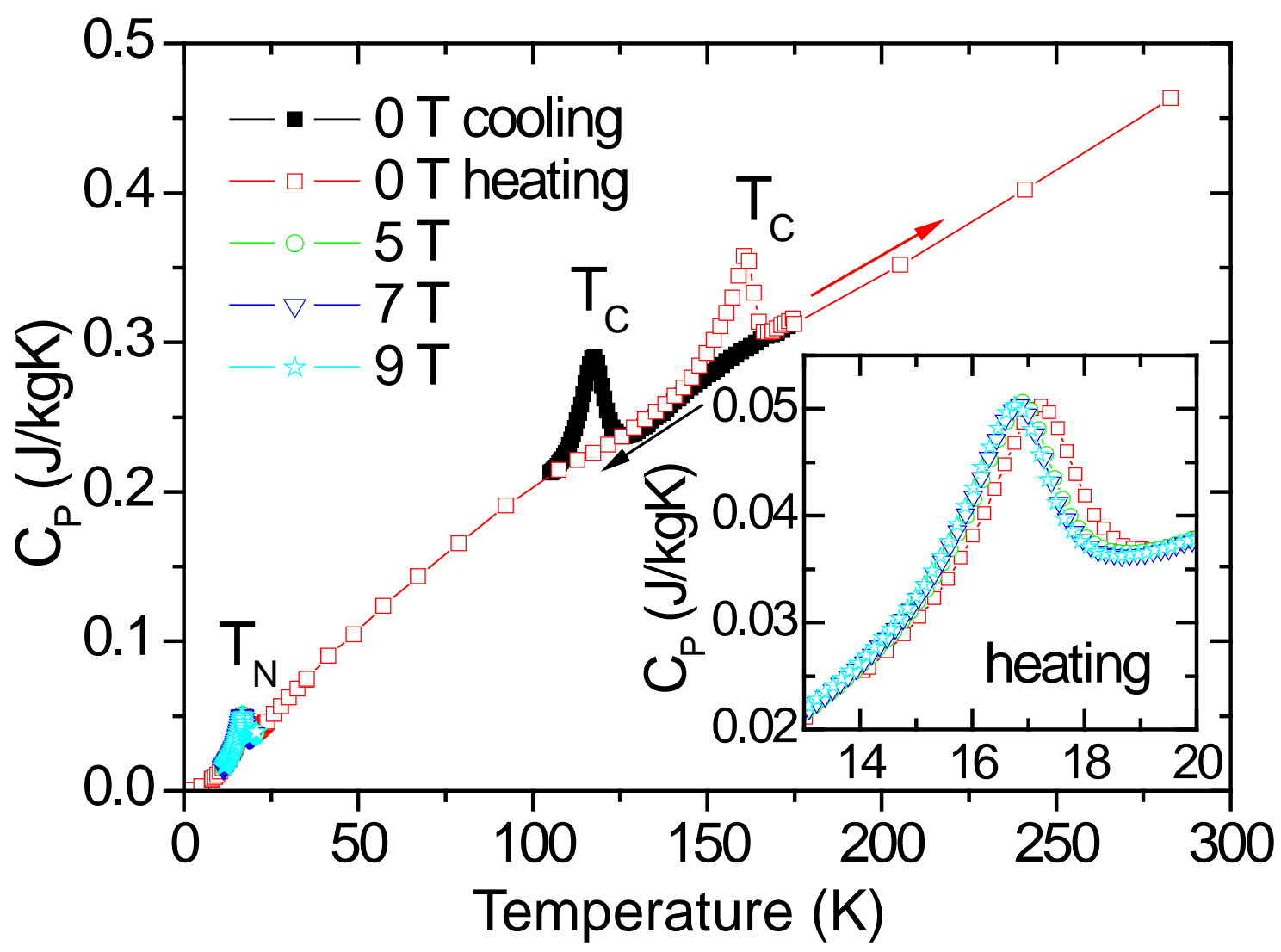

Figure 4 


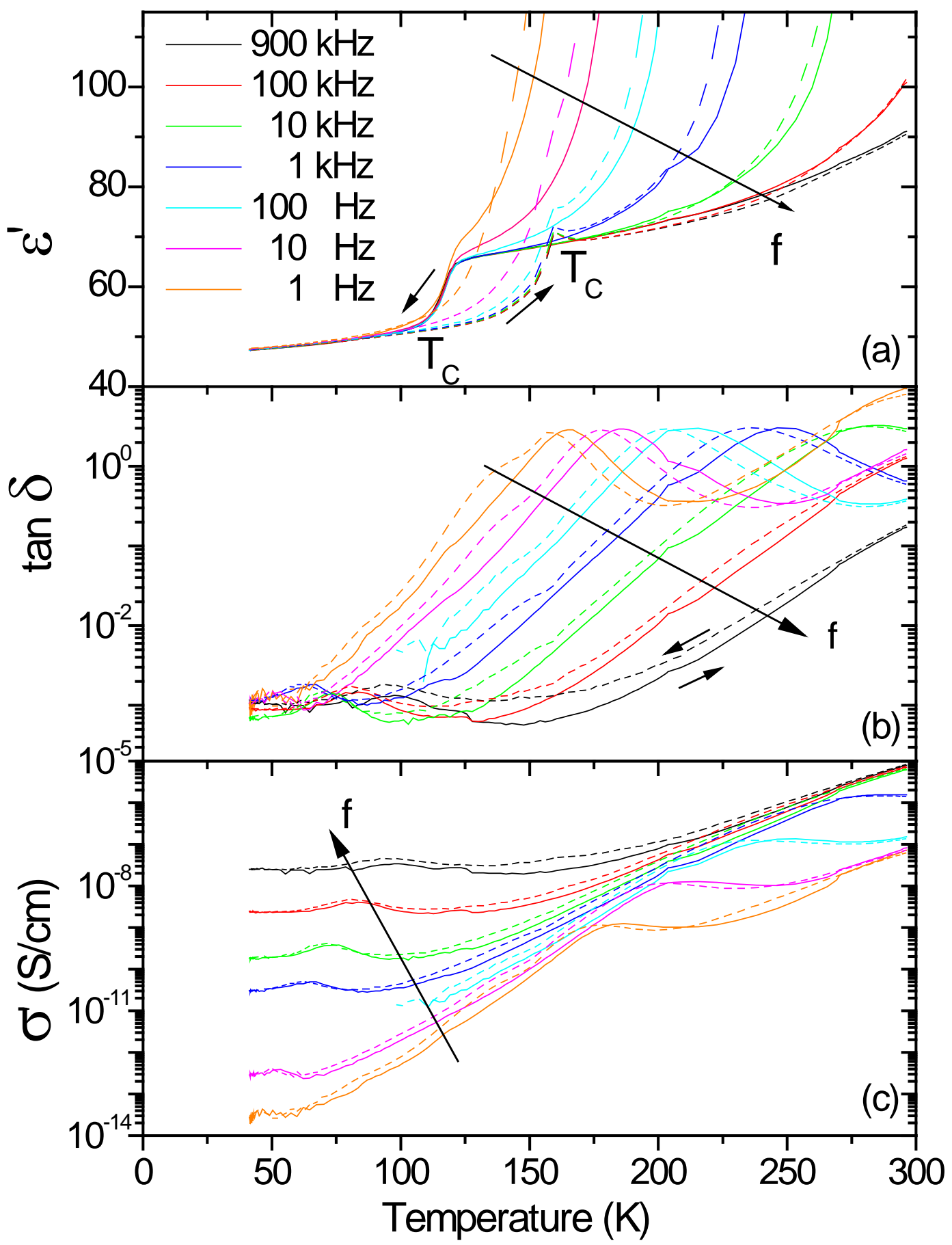

Figure 5 


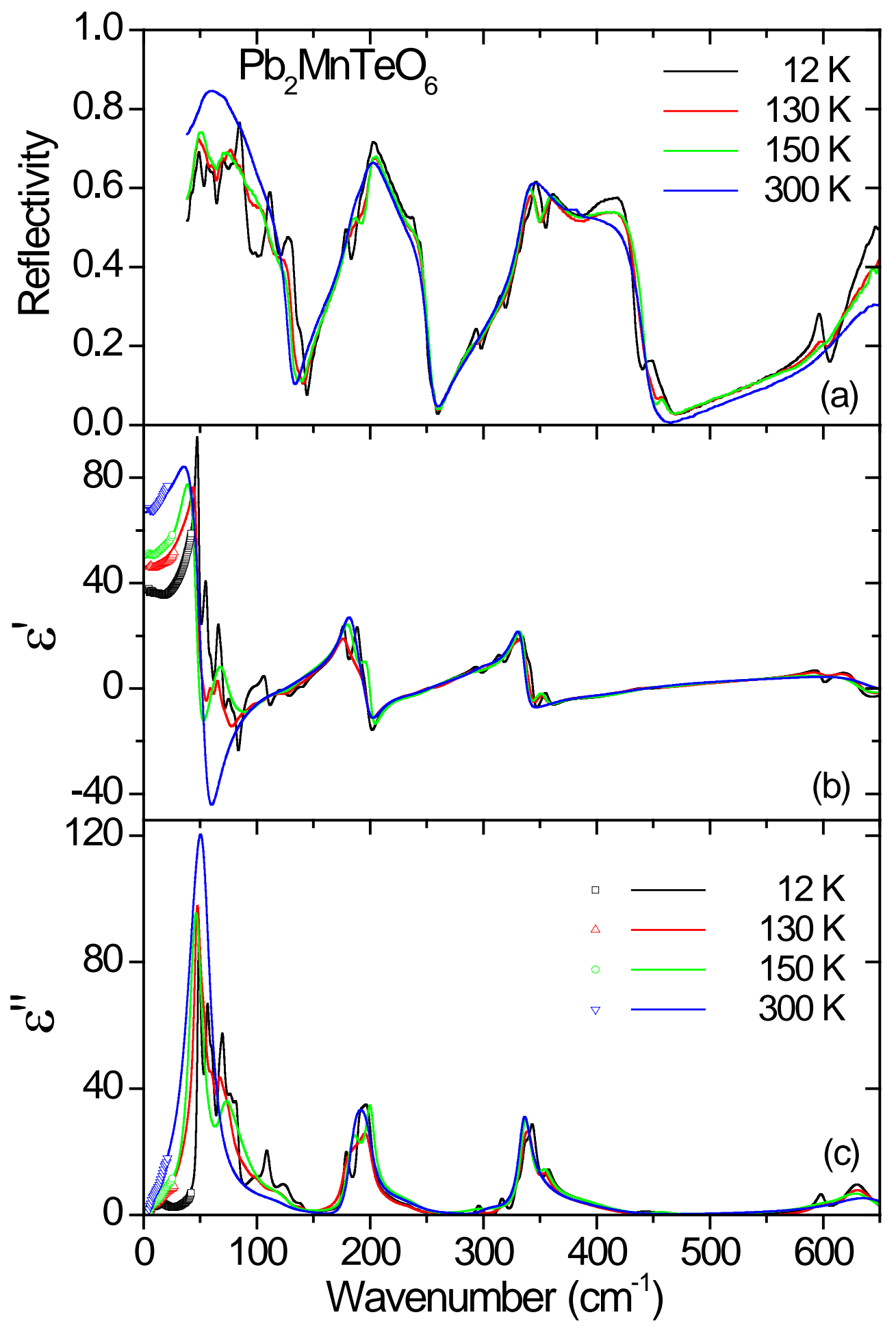

Figure 6 


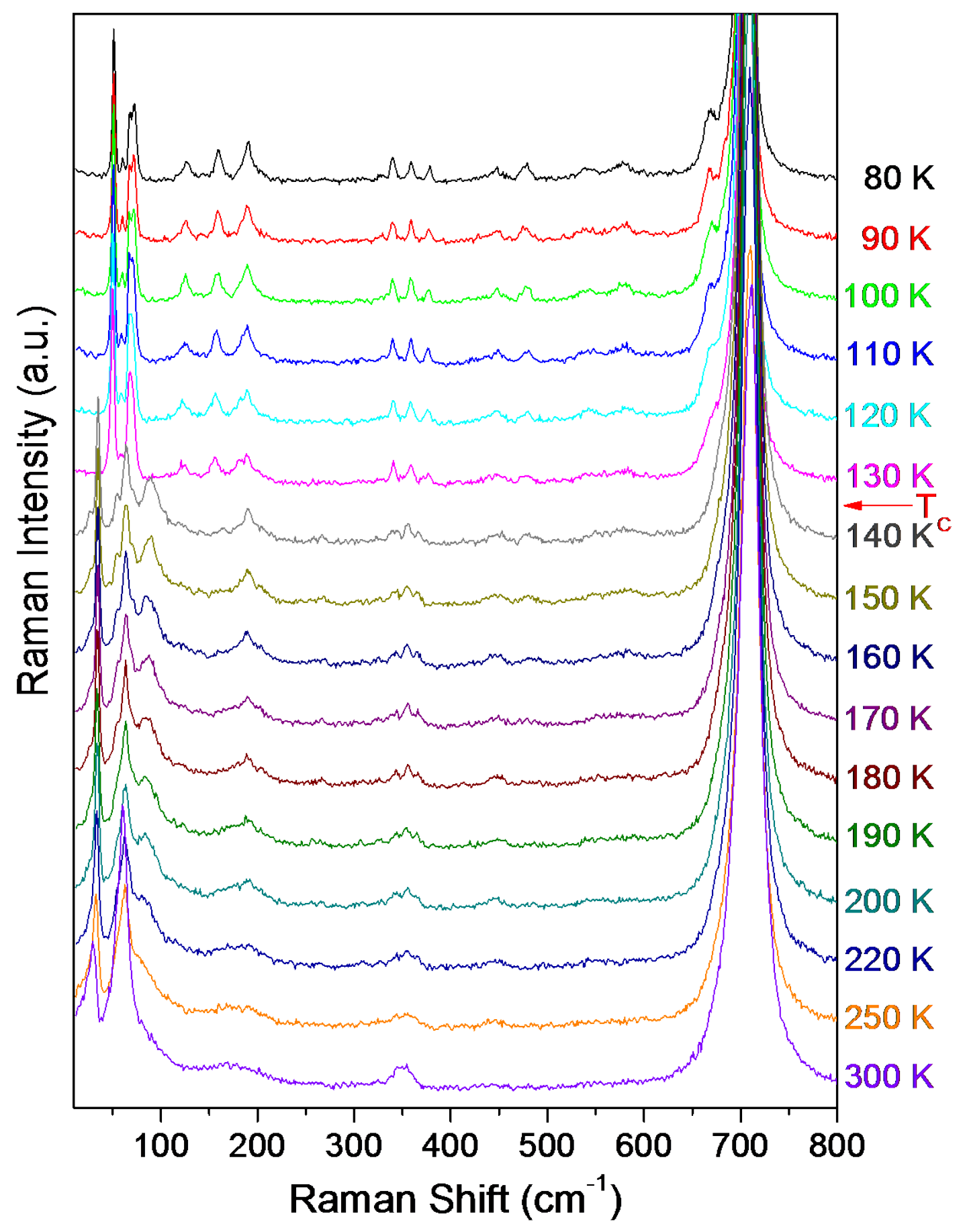

Figure 7 


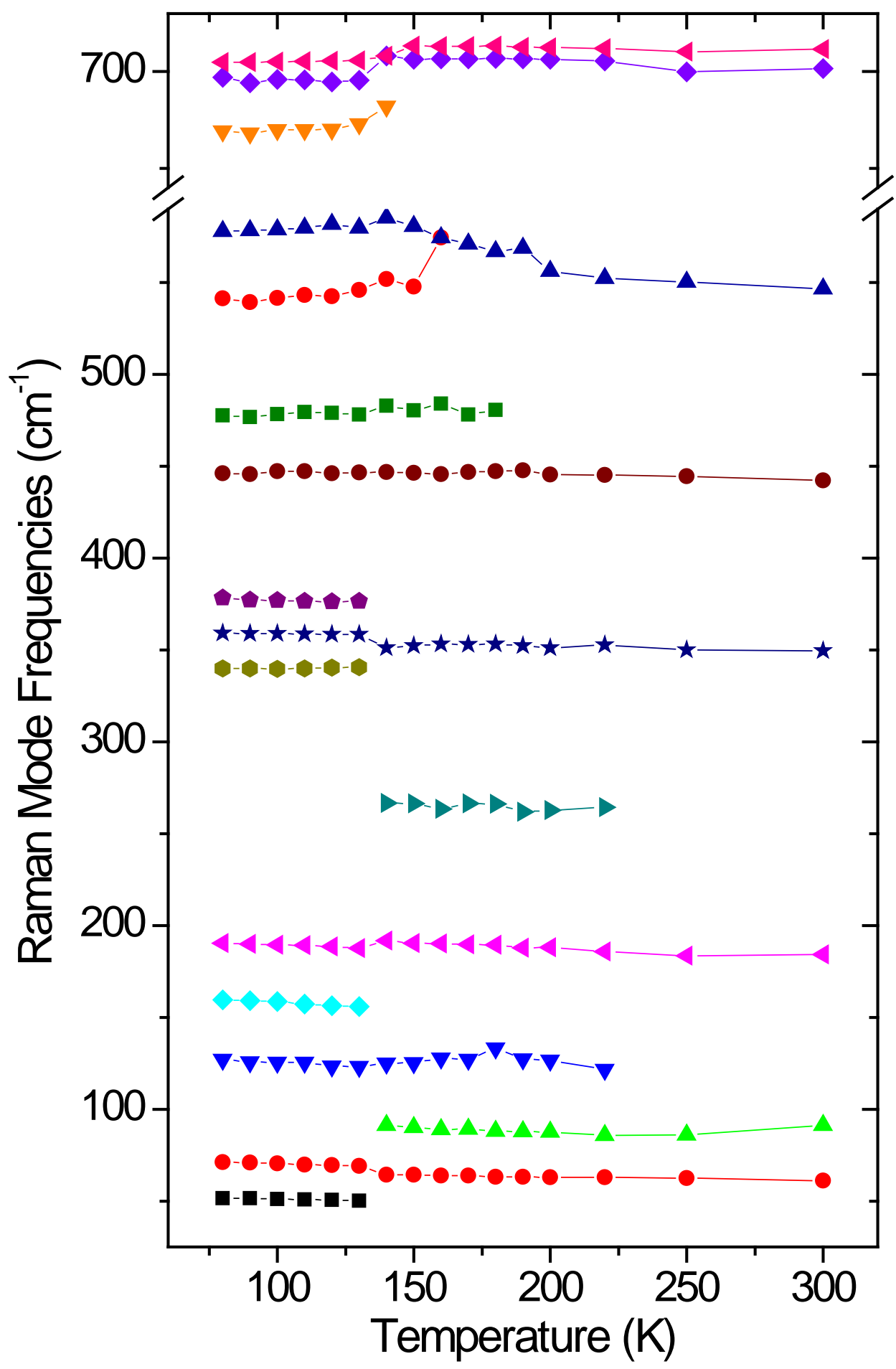

Figure 8 


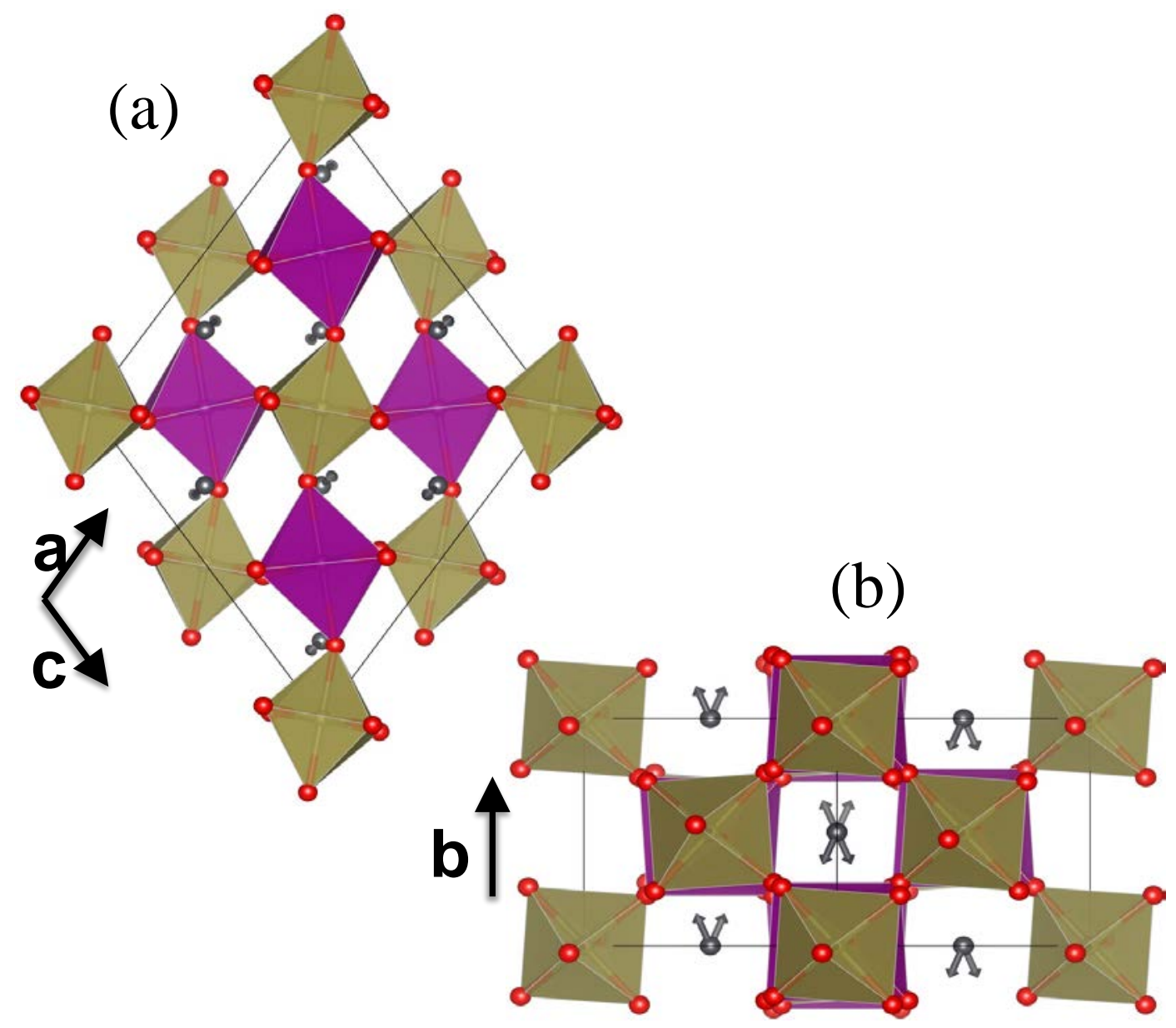

Figure 9 
“For Table of Contents Only”

\section{Table of Contents Graphic and Synopsis}

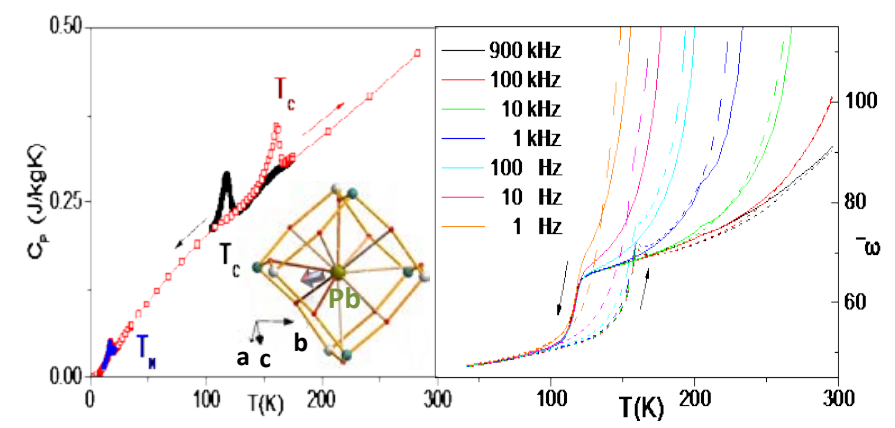

$\mathrm{Pb}_{2} \mathrm{MnTeO}_{6}$ double perovskite presents $I 2 / m$ to $C 2 / c$ phase transition ( $\left.140 \mathrm{~K}\right)$, accompanied by a displacement of $\mathrm{Pb}^{2+}$, together with a surprising off-centering of magnetic $\mathrm{Mn}^{2+} \cdot \mathrm{Pb}_{2} \mathrm{MnTeO}_{6}$ is an antiferromagnet with propagation vector (0 10 ). A jump in dielectric permittivity ( $140 \mathrm{~K})$ suggests antiferroelectricity, however the absence of switching suggests that $\mathrm{Pb}_{2} \mathrm{MnTeO}_{6}$ can only be antipolar. First principle calculations confirmed the crystal and magnetic structures as locally stable, and that antiferroelectric switching is improbable in $\mathrm{Pb}_{2} \mathrm{MnTeO}_{6}$. 